M. A. Kenku and F. Momose

Nagoya Math. J.

Vol. 109 (1988), 125-149

\title{
TORSION POINTS ON ELLIPTIC CURVES DEFINED OVER QUADRATIC FIELDS
}

\author{
M. A. KENKU AND F. MOMOSE
}

Let $k$ be a quadratic field and $E$ an elliptic curve defined over $k$. The authors $[8,12,13][23]$ discussed the $k$-rational points on $E$ of prime power order. For a prime number $p$, let $n=n(k, p)$ be the least non negative integer such that

$$
E_{p^{\infty}}(k)=\bigcup_{m \geqq 0} \operatorname{ker}\left(p^{m}: E \longrightarrow E\right)(k) \subset E_{p^{n}}
$$

for all elliptic curves $E$ defined over a quadratic field $k$ ([15]). For prime numbers $p<300, p \neq 151,199,227$ nor 277 , we know that $n(k, 2)=3$ or $4, n(k, 3)=2, n(k, 5)=n(k, 7)=1, n(k, 11)=0$ or $1, n(k, 13)=0$ or 1 , and $n(k, p)=0$ for all the prime numbers $p \geqq 17$ as above (see loc. cit.). It seems that $n(k, p)=0$ for all prime numbers $p \geqq 17$ and for all quadratic fields $k$. In this paper, we discuss the $N$-torsion points on $E$ for integers $N$ of products of powers of $2,3,5,7,11$ and 13 . Let $N \geqq 1$ be an integer and $m$ a positive divisor of $N$. Let $X_{1}(m, N)$ be the modular curve which corresponds to the finite adèlic modular group

$$
\Gamma_{1}(m, N)=\left\{\left(\begin{array}{ll}
a & b \\
c & d
\end{array}\right) \in \mathrm{GL}_{2}(\hat{Z}) \mid a-1 \equiv c \equiv 0 \bmod N, b \equiv d-1 \equiv 0 \bmod m\right\},
$$

where $\hat{Z}=\varliminf_{n} Z / n Z$. Then $X_{1}(m, N)$ is defined over $\boldsymbol{Q}\left(\zeta_{m}\right)$, where $\zeta_{m}$ is a primitive $m$-th root of 1 . Put $Y_{1}(m, N)=X_{1}(m, N) \backslash$ \{cusps $\}$, which is the coarse moduli space $\left(/ \boldsymbol{Q}\left(\zeta_{m}\right)\right)$ of the isomorphism classes of elliptic curves $E$ with a pair $\left(P_{m}, P_{N}\right)$ of points $P_{m}$ and $P_{N}$ which generate a subgroup $\simeq Z / m Z \times Z / N Z$, up to the isomorphism $(-1)_{E}: E \simeq E$. For $m=1$, let $X_{1}(N)=X_{1}(1, N), \Gamma_{1}(N)=\Gamma_{1}(1, N)$ and $Y_{1}(N)=Y_{1}(1, N)$. For the integers $N=2^{4}, 11$ and $13, X_{1}(N)$ are hyperelliptic and $n(k, 2), n(k, 11)$ and $n(k, 13)$ depend on $k$ [23] (3.3). Our result is the following.

Theorem (0.1). Let $N$ be an integer of a product of powers of 2,3,5,

Received September 29, 1986. 
7, 11 and 13, let $m$ be a positive divisor of $N$. If $X_{1}(m, N)$ is not hyperelliptic (i.e. the genus $g_{1}(m, N) \neq 0$ and $(m, N) \neq(1,11),(1,13),(1,14),(1.15),(1,16)$, $(1,18),(2,10)$ nor $(2,12))$, then $Y_{1}(m, N)(k)=\phi$ for all quadratic fields $k$.

For prime numbers $p \geqq 17$, it seems that $Y_{1}(p)(k)=\phi$ for all quadratic fields $k$ [23]. With Theorem (0.1), we may conjecture that the torsion subgroup of $E(k)$ ( $k=$ a quadratic field) is isomorphic to one of the following groups :

$\begin{array}{llc} & \text { for } 1 \leqq N \leqq 10 \text { or } N=12 & g_{1}(m, N) \\ Z / N Z & \text { for } 1 \leqq n \leqq 4 & 0 \\ Z / 2 Z \times Z / 2 n Z & 0 \\ Z / 3 n \times Z / 3 n Z & \text { for } n=1 \text { or } 2 \text { with } k=Q(\sqrt{-3}) & 0 \\ Z / 4 Z \times Z / 4 Z & \text { with } k=Q(\sqrt{-1}) & 0 \\ Z / N Z & \text { for } N=11,14 \text { or } 16 & 1 \\ Z / N Z & \text { for } N=13,16 \text { or } 18 & 2 \\ Z / 2 Z \times Z / 2 n Z & \text { for } n=5 \text { or } 6 & 1\end{array}$

For $(m, N)=(1,14),(1,15),(1,18),(2,10)$ and $(2,12)$, we give examples of quadratic fields $k$ such that $Y_{1}(m, N)(k)=\phi(2.4)$, (2.5) (see also [23] (3.3)).

The proof of Theorem (0.1) consists of two parts. One is a study on the Mordell-Weil groups of jacobian varieties of some modular curves (1.4), (1.5). The other is a similar discussion as in [8, 12, 13] [23]. Suppose that there is a $k$-rational point $x$ on $Y_{1}(m, N)$ for a pair $(m, N)$ as in $(0.1)$. Then $x$ defines a rational function $g(/ Q)$ on a subcovering $X: X_{1}(m, N) \rightarrow$ $X \rightarrow X_{0}(N)$, whose divisor $(g)$ is determined by $x$. Using the methods as in $[8,12,13][23]$, we show that such a function does not exist and get the result. It will be proved in Section 2 for $m=1$ and in Section 3 for $m \geqq 2$.

Notation. For a rational prime $p, \boldsymbol{Q}_{p}^{u r}$ denotes the maximal unramified extension of $\boldsymbol{Q}_{p}$. Let $K$ be a finite extension of $\boldsymbol{Q}, \boldsymbol{Q}_{p}$ or $\boldsymbol{Q}_{p}^{u r}$, and $A$ an abelian variety defined over $K$. Then $\mathcal{O}_{K}$ denotes the ring of integers of $K$, and $A_{/ O_{K}}$ denotes the Néron model of $A$ over the base $\mathcal{O}_{K}$. For a finite subgroup $G$ of $A$ defined over $K, G_{/_{K}}$ denotes the schematic closure of $G$ in the Néron model $A_{/ O_{K}}$ (, which is a quasi finite flat group scheme [28] $\S 2)$. For a subscheme $Y$ of a modular curve $X / Z$ and for a fixed rational prime $p, Y^{h}$ denotes the open subscheme $Y \backslash$ supersingular points on 
$\left.Y \otimes \boldsymbol{F}_{p}\right\}$. For a finite extension $K$ of $\boldsymbol{Q}$ and for a prime $p$ of $K,\left(\mathcal{O}_{K}\right)_{(p)}$ denotes the local ring at $p$.

\section{§1. Preliminaries}

In this section, we give a review on modular curves and discuss the Mordell-Weil groups of jacobian varieties of some modular curves. Let $N \geqq 1$ be an integer and $m$ a positive divisor of $N$. Let $X_{1}(m, N)$ (resp. $X_{0}(m, N)$ ) be the modular curve $\left(/ \boldsymbol{Q}\left(\zeta_{m}\right)\right)$ (resp. $/ \boldsymbol{Q}$ ) which corresponds to the finite adèlic modular group

$$
\begin{gathered}
\Gamma_{1}(m, N)=\left\{\left(\begin{array}{ll}
a & b \\
c & d
\end{array}\right) \in \mathrm{GL}_{2}(\hat{Z}) \mid a-1 \equiv c \equiv 0 \bmod N, b \equiv d-1 \equiv 0 \bmod m\right\} \\
\left(\operatorname{resp} . \Gamma_{0}(m, N)=\left\{\left(\begin{array}{ll}
a & b \\
c & d
\end{array}\right) \in \mathrm{GL}_{2}(\hat{Z}) \mid c \equiv 0 \bmod N, b \equiv 0 \bmod m\right\}\right) .
\end{gathered}
$$

The modular curve $X_{1}(m, N)$ is the coarse moduli space $\left(/ \boldsymbol{Q}\left(\zeta_{m}\right)\right)$ of the isomorphism classes of the generalized elliptic curves $E$ with a pair $\left(P_{m}, P_{N}\right)$ of points $P_{m}$ and $P_{N}$ which generate a subgroup $\simeq Z / m Z \times Z / N Z$, up to the isomorphism $(-1)_{E}: E \widetilde{\rightarrow} E$ [4]. Let $Y_{1}(m, N), Y_{0}(m, N)$ denote the open affine subschemes $X_{1}(m, N) \backslash\{$ cusps $\}$ and $X_{0}(m, N) \backslash$ cusps $\}$. For $m=1$, let $X_{1}(N)=X_{1}(1, N), X_{0}(N)=X_{0}(1, N), \Gamma_{1}(N)=\Gamma_{1}(1, N), \Gamma_{0}(N)=\Gamma(1, N), Y_{1}(N)$ $=Y_{1}(1, N)$ and $Y_{0}(N)=Y_{0}(1, N)$. Let $K$ be a subfield of $C$. For a $K$ rational point $x$ on $Y_{1}(m, N)$ (resp. $Y_{0}(m, N)$ ), there exists an elliptic curve $E$ defined over $K$ with a pair $\left(P_{m}, P_{N}\right)$ of $K$-rational points $P_{m}$ and $P_{N}$ (resp. $\left(A_{m}, A_{N}\right)$ of cyclic subgroups $A_{m}$ and $A_{N}$ defined over $K$ ) such that (the isomorphism class containing) the pair $\left(E, \pm\left(P_{m}, P_{N}\right)\right.$ ) (resp. the triple $\left.\left(E, A_{m}, A_{N}\right)\right)$ represents $x$ [4] VI (3.2). The modular curve $X_{0}(m N)$ is isomorphic over $\boldsymbol{Q}$ to $X_{0}(m, N)$ by

$$
(E, A) \longmapsto\left(E / A_{N}, A_{N} / A_{N}, E / A_{N}\right),
$$

where $E_{v}=\operatorname{ker}(N: E \rightarrow E)$ and $A_{N}$ is the cyclic subgroup of order $N$ of $A$. Let $\pi=\pi_{m, N}$ be the natural morphism of $X_{1}(m, N)$ to $X_{0}(m, N)$ : $\left(E, \pm\left(P_{m}, P_{N}\right)\right) \mapsto\left(E,\left\langle P_{m}\right\rangle,\left\langle P_{N}\right\rangle\right)$, where $\left\langle P_{m}\right\rangle$ and $\left\langle P_{m}\right\rangle$ are the cyclic subgroups generated by $P_{m}$ and $P_{N}$, respectively. Then $\pi$ is a Galois covering with the Galois group $\bar{\Gamma}_{0}(m, N)=\Gamma_{0}(m, N) / \pm \Gamma_{1}(m, N) \simeq\left((Z / m Z)^{\times} \times\right.$ $\left.(Z / N Z)^{\times}\right) / \pm 1$. For integers $\alpha, \beta$ prime to $N,[\alpha, \beta]$ denotes tha automorphism of $X_{1}(m, N)$ which is represented by $g \in \Gamma_{0}(m, N)$ such that $g \equiv\left(\begin{array}{ll}\beta & 0 \\ 0 & \alpha\end{array}\right)$ $\bmod N$. Then $[\alpha, \beta]$ acts as 


$$
\left(E, \pm\left(P_{m}, P_{N}\right)\right) \longmapsto\left(E, \pm\left(\alpha P_{m}, \beta P_{N}\right)\right) .
$$

When $\alpha \equiv \beta \bmod N$ or $m=1$, let $[\alpha]$ denote $[\alpha, \beta]$. When $m=1$, let $\pi_{N}=\pi_{1, N}$ and $\bar{\Gamma}_{0}(N)=\bar{\Gamma}_{0}(1, N)$. For a positive divisor $d$ of $N$ prime to $N / d$, let $w_{d}$ denote the automorphism of $X_{1}(N)$ defined by

$$
(E, \pm P) \longmapsto\left(E /\left\langle P_{d}\right\rangle, \pm(P+Q) \bmod \left\langle P_{d}\right\rangle\right),
$$

where $P_{d}=(N / d) P$ and $Q$ is a point of order $d$ such that $e_{d}\left(P_{d}, Q\right)=\zeta_{d}$ for a fixed primitive $d$-th root $\zeta_{d}$ of 1 . ( $e_{d}: E_{d} \times E_{d} \rightarrow \mu_{d}$ is the $e_{d}$-pairing). For a subcovering $X: X_{1}(m, N) \rightarrow X \rightarrow X_{0}(N)$ (resp. $X_{1}(N) \rightarrow X \rightarrow X_{0}(N)$ ), we denote also by $[\alpha, \beta]$ (resp. $w_{d}$ ) the automorphism of $X$ induced by $[\alpha, \beta]\left(\right.$ resp. $\left.w_{d}\right)$. For a square free integer $N$, the covering $X_{1}(N) \rightarrow X_{0}(N)$ is unramified at the cusps. Let $\mathscr{X}$ denote the normalization of the projective $j$-line $\mathscr{X}_{0}(1) \simeq P_{Z}^{1}$ in $X$. For $X=X_{1}(m, N), X=X_{0}(m, N), X=X_{1}(N)$ and $X=X_{0}(N)$, let $\mathscr{X}=\mathscr{X}_{1}(m, N), \mathscr{X}=\mathscr{X}_{0}(m, N), \mathscr{X}=\mathscr{X}_{1}(N)$ and $\mathscr{X}=$ $\mathscr{X}_{0}(N)$. Then $\mathscr{X} \otimes Z[1 / N] \rightarrow \operatorname{Spec} Z[1 / N]$ is smooth [4] VI (6.7).

(1.1) Let $\mathbf{0}=\left(\begin{array}{l}0 \\ 1\end{array}\right), \infty=\left(\begin{array}{l}1 \\ 0\end{array}\right)$ be the $\boldsymbol{Q}$-rational cusps on $X_{0}(N)$ which are represented by $\left(\boldsymbol{G}_{m} \times \boldsymbol{Z} / N Z, Z / N Z\right)$ and $\left(\boldsymbol{G}_{m}, \mu_{N}\right)$. Then $w_{N}(\mathbf{0})=\infty$. The cuspidal sections of the fibre $X_{1}(N) \times_{X_{0}(N)} 0$ are represented by the pairs $\left(G_{m} \times Z / N Z, \pm P\right)$ for the points $P \in\{1\} \times Z / N Z$ of order $N$, which are all $\boldsymbol{Q}$-rational. We call them the 0-cusps. For a positive divisor $d$ of $N$ with $1<d<N$ and for an integer $i$ prime to $N$, let $\left(\begin{array}{l}i \\ d\end{array}\right)$ denote the cusps on $X_{0}(N)$ which is represented by $\left(\boldsymbol{G}_{m} \times Z /(N / d) Z, Z / N Z\left(\zeta_{N}, i\right)\right)$, where $Z / N Z\left(\zeta_{N}, i\right)$ is the cyclic subgroup of order $N$ generated by the section $\left(\zeta_{N}, i\right)$. Then $\left(\begin{array}{l}i \\ d\end{array}\right)$ is defined over $Q\left(\zeta_{n}\right)$, where $n=$ G.C.M. of $d$ and $N / d$. When $N$ is a product of $2^{m}$ for $0 \leqq m \leqq 2$ and a square free odd integer, all the cusps on $X_{0}(N)$ are $\boldsymbol{Q}$-rational.

(1.2) Let $\Delta \subset(Z / N Z)^{\times}$be a subgroup containing \pm 1 and $X=X_{\Delta}$ be the modular curve $(/ \boldsymbol{Q})$ corresponding to the modular group

$$
\Gamma_{\Delta}=\left\{\left(\begin{array}{ll}
a & b \\
c & d
\end{array}\right) \in \Gamma_{0}(N) \mid(\operatorname{a} \bmod N) \in \Delta\right\} .
$$

Then $X_{\Delta}$ is the subcovering of $X_{1}(N) \rightarrow X_{0}(N)$ associated with the subgroup $\Delta$. For a prime divisor $p$ of $N$, let $Z^{\prime}$ (resp. $Z$ ) be the irreducible component of the special fibre $\mathscr{X}_{0}(N) \otimes F_{p}$ such that $Z^{\prime h}\left(=Z^{\prime} \backslash\right.$ supersingular points on $\left.\left.\mathscr{X}_{0}(N) \otimes \boldsymbol{F}_{p}\right\}\right)\left(\right.$ resp. $\left.Z^{h}\right)$ is the coarse moduli space $\left(/ \boldsymbol{F}_{p}\right)$ of the 
isomorphism classes of the generalized elliptic curves $E$ with a cyclic subgroup $A, A \simeq Z \mid N Z$ (resp. $A \simeq \mu_{N}$ ), locally for the étale topology ([4] $\mathrm{V}, \mathrm{VI})$. Let $d$ be a positive divisor of $N$ coprime to $N / d$. If $p \mid d$, then $w_{d}$ exchanges $Z^{\prime}$ with $Z$. If $p \nmid d$, then $w_{d}$ fixes $Z^{\prime}$ and $Z$. Let $Z_{X}^{\prime}$ be the fibre $\mathscr{X} \times_{x_{0}(N)} Z^{\prime}$. Then $Z_{X}^{\prime n}$ is smooth over $F_{p}$ and the 0 -cusps $\left(\otimes F_{p}\right)$ are the sections of $Z_{X}^{\prime h}$. If $p \| N$ and $\Delta$ contains the subgroup

$$
\left\{a \in(Z / N Z)^{\times} \mid(a \bmod N / p)= \pm 1\right\},
$$

then $\mathscr{X} \otimes F_{p}$ is reduced and $\mathscr{X}^{h} \otimes Z_{(p)} \rightarrow \operatorname{Spec} Z_{(p)}$ is smooth, where $Z_{(p)}$ is the localization of $Z$ at $(p)$ ([4] VI).

(1.3) We will make use of the following subcoverings $X=X_{4}: X_{1}(m N)$ $\rightarrow X \rightarrow X_{\jmath}(m N)$.

\begin{tabular}{ccccc}
$m$ & $N$ & \multicolumn{1}{c}{$X$} & 4 & genus of $X$ \\
1 & 14 & $X=X_{1}(14) \stackrel{3}{\longrightarrow} X_{0}(14)$ & $\{ \pm 1\}$ & 1 \\
1 & 15 & $X=X_{1}(15) \stackrel{4}{\longrightarrow} X_{0}(15)$ & $\{ \pm 1\}$ & 1 \\
1 & 18 & $X=X_{1}(18) \stackrel{3}{\longrightarrow} X_{0}(18)$ & $\{ \pm 1\}$ & 2 \\
1 & 20 & $X=X_{1}(20) \stackrel{4}{\longrightarrow} X_{0}(20)$ & $\{ \pm 1\}$ & 3 \\
1 & 21 & $X_{1}(21) \stackrel{2}{\longrightarrow} X \stackrel{3}{\longrightarrow} X_{0}(21)$ & $(Z / 3 Z)^{\times} \times\{ \pm 1\}$ & 3 \\
1 & 24 & $X_{1}(24) \stackrel{2}{\longrightarrow} X \stackrel{2}{\longrightarrow} X_{0}(24)$ & $(Z / 3 Z)^{\times} \times\{ \pm 1\}$ & 3 \\
1 & 35 & $X_{1}(35) \stackrel{4}{\longrightarrow} X \stackrel{3}{\longrightarrow} X_{0}(35)$ & $(Z / 5 Z)^{\times} \times\{ \pm 1\}$ & 7 \\
1 & 55 & $X_{1}(55) \stackrel{10}{\longrightarrow} X \stackrel{2}{\longrightarrow} X_{0}(55)$ & $\{ \pm 1\} \times(Z / 11 Z)^{\times}$ & 9 \\
2 & 16 & $X_{1}(32) \stackrel{2}{\longrightarrow} X=X_{1}(2,16) \stackrel{8}{\longrightarrow} X_{0}(32)$ & $\{ \pm(1+16)\}$ & 5 \\
2 & 10 & $X_{1}(20) \stackrel{2}{\longrightarrow} X=X_{1}(2,10) \stackrel{2}{\longrightarrow} X_{0}(20)$ & $\{ \pm 1\} \times\{ \pm 1\}$ & 1 \\
2 & 12 & $X_{1}(24) \stackrel{2}{\longrightarrow} X=X_{1}(2,12) \stackrel{2}{\longrightarrow} X_{0}(24)$ & $\{ \pm 1\} \times\{ \pm 1\}$ & 1
\end{tabular}

(1.4) Mordell-Weil group of $J(X)$.

Let $J_{1}(m, N)$ and $J_{0}(m, N)$ be the jacobian varieties of $X_{1}(m, N)$ and $X_{0}(m, N)$, respectively. For $m=1, J_{1}(1, N)=J_{1}(N)$ and $J_{0}(1, N)=J_{0}(N)$. For the integers $N=13 q, q=2,3,5$ and 11, there exist (optimal) quotients $(/ \boldsymbol{Q})$ of $J_{0}(N)$ whose Mordell-Weil groups are of finite order ([36] table 1,5). For $m=1$ and $N=14,15,18,20,21,24,35$ and 55 , and $(m, N)=$ 
$(2,10),(2,12)$, let $X=X_{4}$ be the subcoverings in (1.3) and $J(X)$ be their jacobian varieties. Then $J_{1}(2,10)$ and $J_{1}(2,12)$ are elliptic curves with finite Mordell-Weil groups ([36] table 1). Let Coker $\left(J_{0}(N) \rightarrow J(X)\right.$ ) be the cokernels of the morphisms as the Picard varieties. In the following table, the factors $A(\mid Q)$ of $J(X)$ have finite Mordell-Weil groups ([36] table 1, 5, [8] [14] [19], (1.5) below).

$\begin{array}{lccc}N & \text { factor } A \text { of } J(X) \text { or } A=J_{0}(N) & \operatorname{dim} A & \text { genus of } X_{0}(N) \\ 22 & J_{0}(22) & 2 & 2 \\ 33 & J_{0}(33) & 3 & 3 \\ 55 & \text { Coker }\left(J_{0}(55) \longrightarrow J(X)\right) & 4 & 5 \\ 77 & J_{0}(77) /\left(1+w_{11}\right) J_{0}(77) & 3 & 7 \\ 14 & J_{1}(14) & 1 & 1 \\ 21 & \text { Coker }\left(J_{0}(21) \longrightarrow J(X)\right) & 3 & 1 \\ 28 & J_{0}(28) & 2 & 2 \\ 35 & \text { Coker }\left(J_{0}(35) \longrightarrow J(X)\right) & 4 & 3 \\ 20 & J_{1}(20) & 3 & 1 \\ 30 & J_{0}(30) & 3 & 3 \\ 45 & J_{0}(45) & 3 & 3 \\ 24 & \text { Coker }\left(J_{0}(24) \longrightarrow J(X)\right) & 3 & 1 \\ 15 & J_{1}(15) & 1 & 1 \\ 18 & J_{1}(18) & 2 & 0 \\ 36 & J_{0}(36) & 1 & 1 \\ 72 & J_{0}(72) & 5 & 5 \\ 32 & J_{0}(32) & 1 & 1 \\ 27 & J_{0}(27) & 1 & 1 \\ 10 & J_{1}(2,10) & 1 & 1 \\ 12 & J_{1}(2,12) & 1 & 1 \\ 16 & J_{1}(2,16) & 5 & 1\end{array}$

Proposition (1.5). For the integers $N=20,21,24,35$ and 55, let $X=$ $X_{4}$ be the subcoverings in (1.3) and put $C_{X}=\operatorname{Coker}\left(J_{0}(N) \rightarrow J(X)\right)$. Then $\# C_{X}(\boldsymbol{Q})<\infty$.

Proof.

Case $N=20$ : We use a result of Coates-Wiles on the Mordell-Weil groups of elliptic curves with complex multiplication ([1] [3] [29]). Let $\chi$ 
be the multiplicative character of $(Z[\sqrt{-1}] /(2+\sqrt{-1}))^{\times}$with $\chi(\sqrt{-1})=$ $-\sqrt{-1}$, and put

$$
\varepsilon=\left(\frac{-1}{}\right) \cdot \chi_{\mid(Z / 5 Z) \times} \text { and } \bar{\varepsilon}=\left(\frac{-1}{}\right) \cdot \chi_{\mid(Z / 5 Z) \times}^{-1},
$$

where $(-1)$ is the quadratic residue symbol. Let $f_{\varepsilon}, f_{i}$ be the new forms ([2]) belonging to $S_{2}\left(\Gamma_{1}(20)\right.$ ) (= the $C$-vector space of holomorphic cusp forms of weight 2 belonging to $\Gamma_{1}(20)$ ) which are associated with the neben types characters $\varepsilon$ and $\bar{\varepsilon}$, respectively; Let $\psi$ be the primitive Grössen character of $\boldsymbol{Q}(\sqrt{-1})$ with conductor $(2+\sqrt{-1})$ such that $\psi((\alpha))=\chi(\alpha) \alpha$ for $\alpha \in \boldsymbol{Q}(\sqrt{-1})^{\times}$prime to the conductor $(2+\sqrt{-1})$. Then

$$
f_{\varepsilon}(z)=\sum \psi(\mathfrak{U}) \exp (2 \pi \sqrt{-1} N(\mathfrak{U}) z),
$$

where $N(\mathfrak{U})=N_{Q(\sqrt{-1}) / Q}(\mathfrak{U})$ is the norm of the ideal $\mathfrak{U} \neq\{0\}$ and $\mathfrak{A}$ runs over the set of integral ideals of $\boldsymbol{Q}(\sqrt{-1})$ ([33]). The modular curve $X_{1}(20)$ is of genus 3 and $H^{0}\left(X_{1}(20) \otimes C, \Omega^{1}\right)=H^{0}\left(X_{0}(20) \otimes C, \Omega^{1}\right) \oplus C f_{\varepsilon} d z \oplus C f_{\bar{\varepsilon}} d z$. For a cusp form $f \in S_{2}\left(\Gamma_{1}(20)\right)$ and $g=\left(\begin{array}{ll}a & b \\ c & d\end{array}\right) \in \mathrm{GL}_{2}^{+}(\boldsymbol{Q})$, put

$$
f \mid[g]_{2}(z)=(a d-b c)(c z+d)^{-2} f\left(\frac{a z+b}{c z+d}\right) \text { and } f \mid K(z)=(f(-\bar{z}))^{-},
$$

where - is the complex conjugation. Then for $H=\left[\left(\begin{array}{lr}0 & -1 \\ 20 & 0\end{array}\right)\right]_{2}, f_{\varepsilon} \mid H=$ $\lambda f_{\bar{\varepsilon}}$ with the absolute value $|\lambda|=1$ ([2]). Put $g=f_{\varepsilon}-f_{\varepsilon} \mid H$ and $h=f_{\varepsilon}+f_{\varepsilon} \mid H$. Then $g=f_{\varepsilon}+e^{-2 \sqrt{-1} \theta} f_{\varepsilon} \mid K=e^{-\sqrt{-1} \theta}\left(e^{\sqrt{-1} \theta} f_{\varepsilon}+e^{\sqrt{-1} \theta} f_{\varepsilon} \mid K\right)$ for a real number $\theta$, and $e^{\sqrt{-1} \theta} g$ is real on the pure imaginary axis ([24] §2). $C_{X}=\operatorname{Coker}\left(J_{0}(20)\right.$ $\rightarrow J(X))$ is isogenous over $\boldsymbol{Q}(\sqrt{-1})$ to the product of two elliptic curves $E_{\varepsilon}$ and $E_{\bar{\varepsilon}}$ with $H^{0}\left(E_{\varepsilon} \otimes C, \Omega^{1}\right)=C f_{\varepsilon} d z$ and $H^{0}\left(E_{\bar{\varepsilon}} \otimes C, \Omega^{1}\right)=C f_{\bar{\varepsilon}} d z$. Further $C_{X}$ is isogenous over $\boldsymbol{Q}$ to the restriction of scalars $\operatorname{Re}_{\boldsymbol{Q}(\sqrt{-1}) / \boldsymbol{Q}}\left(E_{\varepsilon / \boldsymbol{Q}(\sqrt{-1})}\right)$ ([5] [34]). For a cusp form $f \in S_{2}\left(\Gamma_{1}(20)\right)$, put

$$
(2 \pi / \sqrt{20})^{-s} \Gamma(s) L_{f}(s)=\int_{0}^{\infty} t^{s} f(\sqrt{-1} t / \sqrt{20}) \frac{d t}{t}
$$

and

$$
I(f)=\int_{0}^{\infty} f(\sqrt{-1} t / \sqrt{20}) d t .
$$

The (1-dimensional) $L$-function of $C_{X} / \boldsymbol{Q}$ and that of $E_{\varepsilon} / \boldsymbol{Q}(\sqrt{-1})$ are $L_{f_{s}}(s) L_{f_{s}}(s)$ and $L_{f_{\varepsilon}}(1) L_{f_{s}}(1)=\left|L_{f_{s}}(1)\right|^{2}$ (, since $\left.f_{\bar{s}}=f_{\varepsilon} \mid K\right)$ ([21]). The rank of $C_{X}(\boldsymbol{Q})$ is zero if and only if $E_{\varepsilon}(\boldsymbol{Q} \sqrt{-1})<\infty$. Then by the result on the Birch-Swinnerton Dyer conjecture for elliptic curves with complex multi- 
plication ([1] [3] [29]), it suffices to show that $I\left(f_{\varepsilon}\right) \neq 0$. One sees that $I(h)=0$ and $I\left(f_{\varepsilon}\right)=\frac{1}{2}(I(g)+I(h))$. Since $e^{\sqrt{-1} \theta} g$ is real on the pure imaginary axis, it suffices to show that $g(\sqrt{-1} t / \sqrt{20}) \neq 0$ for all $t>0$. Let $\gamma=\left(\begin{array}{ll}a & b \\ c & d\end{array}\right) \in \Gamma_{0}(20)$ with $\varepsilon(a)=-1$. The $g\left|[\gamma]_{2}=-g=g\right| H$, hence for $\delta=$ $\gamma^{-1}\left(\begin{array}{lr}0 & -1 \\ 20 & 0\end{array}\right), g \mid[\delta]_{2}=g$. The quotient $X_{1}(20) /\langle\delta\rangle$ is an elliptic curve, so the zero points of $g d z$ are the fixed points of $\delta$. The automorphism $\delta$ has four fixed points, which correspond to $(-20 \beta+\sqrt{-20}) / 20 \alpha$ for integers $\alpha$ and $\beta$ such that $\varepsilon(\alpha)=-1$ and $\left(\begin{array}{ll}\alpha & \beta \\ * & *\end{array}\right) \in \Gamma_{0}(20)$. Then $\beta \neq 0$, so $\delta$ does not have the fixed points on the pure imaginary axis.

For the remaining cases for $N=21,24,35$ and 55, we apply a Mazur's method in [14] [19]. It suffices to show that $C_{X}$ is $\boldsymbol{Q}$-simple and that $C_{X}(\boldsymbol{Q})$ has a subgroup $\neq\{0\}$ of order prime to the class numbers of $\boldsymbol{Q}\left(\zeta_{N}\right)$, where $\zeta_{N}$ is a primitive $N$-th root of 1 (see loc. cit.). For the class numbers, see e.g. [6] table.

Case $N=21$ and 24: $C_{X}$ are $\boldsymbol{Q}$-simple. By [35], one finds cuspidal subgroups of order $13(N=21)$ and $5(N=24)$.

Case $N=35$ : The characteristic polynomial of the Hecke operator $T_{2}$ on $S_{2}\left(\Gamma_{4}\right)$ (associated with the prime number 2) is

$$
\left(X^{3}+X^{2}-4 X\right) \times\left(X^{4}+2 X^{3}-7 X^{2}-14 X+1\right) .
$$

The first factor of the above polynomial corresponds to $X_{0}(35)$, so $C_{X}$ is $\boldsymbol{Q}$-simple. There is a cuspidal subgroup of order 13 (see loc. cit.).

Case $N=55$ : The characteristic polynomial of $T_{2}$ on $S_{2}\left(\Gamma_{4}\right)$ is

$$
(X+2)^{2}(X-1)\left(X^{2}-2 X-1\right) \times\left(X^{4}-9 X^{2}+12\right) \text {. }
$$

$C_{X}$ corresponds to $X^{4}-9 X^{2}+12$ ([36] table 5), so $C_{X}$ is $Q$-simple. There is a cuspidal subgroup of order 3 .

(1.6) The following curves are hyperelliptic (of genus $\geqq 2$ ).

$\begin{array}{lc}\text { curve } & \text { hyperelliptic involution } \\ X_{1}(18) & w_{2}[5] \\ X_{0}(22) & w_{22} \\ X_{0}(33) & w_{11} \\ X_{0}(28) & w_{7} \\ X_{0}(30) & w_{15} \\ X_{1}(13) & {[5]}\end{array}$


Proposition (1.7) ([7], [8]). Let $X$ be the subcoverings in (1.3) for $(m, N)$ $=(2,16),(1,20),(1,21),(1,24)$ and $(1,35)$. Then $X$ are not hyperelliptic.

(1.8) For $N=35,55$ (resp. 77), let $X$ be the subcoverings in (1.3) (resp. $X=X_{0}(77)$ ). For an automorphism $\gamma$ of $X$, let $S_{\gamma}$ denote the number of the fixed points of $\gamma$. Then we see the following.

$\begin{array}{lcr}N & \gamma & S_{r} \\ 35 & \left(E, A_{5}, \pm P_{7}\right) \longmapsto\left(E / A_{5}, E_{5} / A_{5}, \pm 3 P_{7} \bmod A_{5}\right) & 12 \\ 55 & \left(E, \pm P_{5}, A_{11}\right) \longmapsto\left(E / A_{11}, \pm 2 P_{5} \bmod A_{11}, E_{11} / A_{11}\right) & 16 \\ 77 & \gamma=w_{77}:(E, A) \longmapsto\left(E / A, E_{77} / A\right) & 8\end{array}$

Here $P_{m}$ is a point of order $m$ and $A_{m}$ is a subgroup of order $m$.

For the integers $N$ in (1.8), we will apply the following lemma.

Lemma (1.9). Let $K$ be a field, $X$ a proper smooth curve defined over $K$ and $(1 \neq) \gamma$ an automorphism of $X$ with the fixed points $x_{i}, 1 \leqq i \leqq s$. Let $f$ be a rational function on $X$ such that the divisors $\left(\gamma^{*} f\right) \neq(f)$. Then the degree of $f \leqq s / 2$ and

$$
\left(\gamma^{*} f / f-1\right)_{0}>\Sigma^{\prime}\left(x_{i}\right)
$$

where $\sum^{\prime}$ is the sum of the divisors $\left(x_{i}\right)$ such that $f\left(x_{i}\right) \neq 0, \infty$.

Proof. Let $S_{0}$ (resp. $S_{\infty}$, resp. $T$ ) be the set of the fixed points of $\gamma$ consisting of $x_{i}$ with $f\left(x_{i}\right)=0$ (resp. $f\left(x_{i}\right)=\infty$, resp. $x_{i} \notin S_{0} \cup S_{\infty}$ ). Then the divisor

$$
(f)=E+\sum_{x_{i} \in S_{0}} n_{i}\left(x_{i}\right)-F-\sum_{x_{i} \in S_{\infty}} n_{i}\left(x_{i}\right),
$$

for effective divisors $E$ and $F$, and positive integers $n_{i}$. Then

$$
\left(\gamma^{*} f / f\right)=\gamma^{*} E+F-E-\gamma^{*} F .
$$

By the assumption $\left(\gamma^{*} f\right) \neq(f), g=\gamma^{*} f / f$ is not a constant function, so $\operatorname{deg}(g) \leqq 2 \cdot \operatorname{deg}(f)-\sum_{x_{i} \in S_{0} \cup S_{\infty}} n_{i}$. For $x_{i} \in T, g\left(x_{i}\right)=1$. Therefore

$$
(g-1)_{0}>\sum_{w_{i} \in T}\left(x_{i}\right) \text {. }
$$

Then $\operatorname{deg}(g) \geqq \# T . \quad$ Further $2 \cdot \operatorname{deg}(f) \geqq \operatorname{deg}(g)+\sum_{x_{i} \in S_{0} \cup S_{\infty}} n_{i} \geqq s$.

Proposition (1.10) ([28] (3.3.2) [27]). Let $K$ be a finite extension of $\boldsymbol{Q}_{p}^{u r}$ of degree $e \leqq p-1$ with the ring of integers $R=\mathcal{O}_{K} . \quad$ Let $G_{i}(i=1,2)$ be finite flat group schemes over $R$ of rank $p$ and $f: G_{1} \rightarrow G_{2}$ be a homomorphism such that $f \otimes K: G_{1} \otimes K \rightarrow G_{2} \otimes K$ is an isomorphism. If $e<$ 
$p-1$, then $f$ is an isomorphism. If $e=p-1$ and $f$ is not an isomorphism, then $G_{1} \simeq(Z / p Z)_{/ R}$ and $G_{2} \simeq \mu_{p / R}$.

Corollary (1.11). Under the notation as in (1.10), assume that $e<$ $p-1$. Let $G$ be a finite flat group scheme over $R$ of rank $p$ and $x$ an $R$ section of $G$. If $x \otimes \overline{\boldsymbol{F}}_{p}=0$ (= the unit section), then $x=0$.

(1.12) Let $K$ be a finite extension of $\boldsymbol{Q}_{p}$ with the ring of integers $R=\mathcal{O}_{K}$ and its residue field $\simeq F_{q}$. Put $N=N^{\prime} \cdot p^{r}$ for the integer $N^{\prime}$ prime to $p$. We here set an assumption on $N$ that $r=0$ if the absolute ramification index $e$ of $p$ (in $K) \geqq p-1$. Let $E$ be an elliptic curve defined over $K$ with a finite subgroup $G \subset E(K)$ of order $N$. Then by the universal property of the Néron model, the schematic closure $G_{/ R}$ of $G$ in $E_{/ R}$ is a finite étale subgroup scheme (, since $e<p-1$ if $r>0$ (1.11)). If $N \neq 2,3$ nor 4 , then $E_{/ R}$ is semistable (see e.g. [36] p. 46). When $E$ has good reduction, the Frobenius map $F=F_{q}: E_{/ R} \otimes F_{q} \rightarrow E_{/ R} \otimes F_{q}$ acts trivially on $G_{/ R} \otimes F_{q}$. In particular, $N \leqq(1+\sqrt{q})^{2}$ (by the Riemann-Weil condition). When $E$ has multiplicative reduction, the connected component $T$ of $E_{/ R} \otimes F_{q}$ of the unit section is a torus such that $T\left(F_{q}\right) \simeq Z /(q-\varepsilon) Z$ for $\varepsilon= \pm 1$. For a prime divisor $l$ of $N$, the $l$-primary part of $G\left(F_{q}\right) \simeq$ $Z / l^{s} \boldsymbol{Z} \times \boldsymbol{Z} / l^{t} \boldsymbol{Z}$ for integers $s, t$ with $0 \leqq s \leqq t$. Then $l^{s}$ divides $q-\varepsilon$ and $E_{/ R} \otimes F_{q}$ contains $T \times Z / l^{s} Z$. If $l^{t} \nmid q-\varepsilon$, then $E_{/ R} \otimes F_{q}$ contains $T \times Z / l^{t} Z$.

(1.13) Let $X\left(\rightarrow X_{0}(1)\right)$ be a modular curve defined over $\boldsymbol{Q}$ with its jacobian variety $J=J(X)$. Let $k$ be a quadratic field and $p$ be a prime of $k$ lying over a rational prime $p$. Let $R=\left(\mathcal{O}_{k}\right)_{(p)}, Z_{(p)}$ denote the localizations at $p$ and $p$, respectively. Let $x$ be a $k$-rational point on $X$ such that $x \otimes \kappa(p)$ is a section of the smooth part $\mathscr{X}^{\text {smooth }} \otimes Z_{(p)}$ and that $X \otimes \kappa(p)$ $=C \otimes \kappa(p), \quad x^{\sigma} \otimes \kappa(p)=C_{\sigma} \otimes \kappa(p)$ for $Q$-rational cusps $C, C_{\sigma}$ and $1 \neq$ $\sigma \in \operatorname{Gal}(k / Q)$, where $\mathscr{X}$ is the normalization of the projective $j$-line $\mathscr{X}_{0}(1)$ $\simeq \boldsymbol{P}_{Z}^{1}$ in $X$. Consider the $\boldsymbol{Q}$-rational section $i(x)=\operatorname{cl}\left((x)+\left(x^{\sigma}\right)-(C)-\left(C_{\sigma}\right)\right)$ of the Néron model $J_{/ Z}$ :

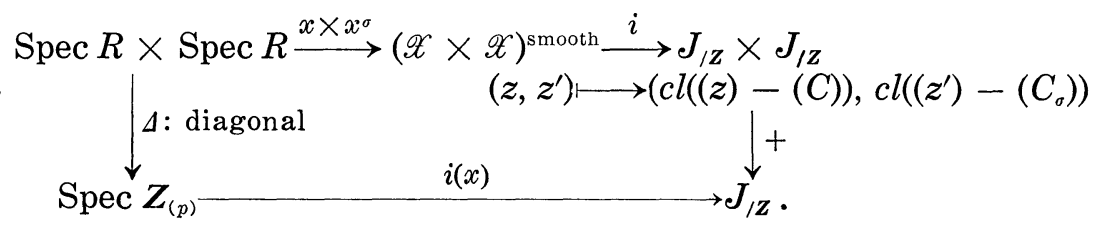

Then $\left(\left(x \times x^{\sigma}\right) \cdot i \cdot+\right) \otimes \kappa(p)=0$ (= the unit section), hence $i(x) \otimes F_{p}=0$. 
Let $A / \boldsymbol{Q}$ be a quotient of $J ; J \stackrel{j}{\longrightarrow} A$ which has the Mordell-Weil group of finite order. If $p \neq 2$, then the specialization Lemma (1.11) shows that $j \cdot i(x)=0$.

Remark (1.14). Under the notation as in (1.13), wa here consider the case when $C$ and $C_{\sigma}$ are not $Q$-rational. Assume that the set $\left\{C, C_{\sigma}\right\}$ is $\boldsymbol{Q}$-rational and that $C \otimes \boldsymbol{Z}_{(p)}$ and $C_{\sigma} \otimes \boldsymbol{Z}_{(p)}$ are the sections of $\mathscr{C}^{\text {smooth }} \otimes \boldsymbol{Z}_{(p)}$. Let $K$ be the quadratic field over which $C$ and $C_{\sigma}$ are defined. Let $p^{\prime}$ be a prime of $K$ lying over $p$ and $e^{\prime}$ be the ramification index $p$ in $K$. Then by the same way as in (1.3), we get $i(x) \otimes \kappa\left(p^{\prime}\right)=0$ in $J_{1 K_{K}}$. If $e^{\prime}<p-1$ or $p$ does not divide $\sharp A(\boldsymbol{Q})$, then $j \cdot i(x)=0$.

For a finite extension $K$ of $\boldsymbol{Q}$ and for an abelian variety $A$ defined over $K$, let $f(A / K)$ denote the conductor of $A$ over $K$.

Lemma (1.15) ([21] Proposition 1). Let E be an elliptic curve defined over a finite extension $K$ of $\boldsymbol{Q}$ and $L$ be a quadratic extension of $K$, with the relative discriminant $D=D(L / K)$. Then the restriction of scalars $\operatorname{Re}_{L / K}\left(E_{/ L}\right)$ ([5] [34]) is isogenous over $K$ to a product of $E$ and an elliptic curve $F(\mid K)$ with $f(E / K) f(F / K)=N_{L / K}(f(E / L))^{2} D$.

\section{§2. Rational points on $X_{1}(N)$}

Let $k$ be a quadratic field and $N$ an integer of a product of $2,3,5$, 7, 11 and 13. Let $x$ be a $k$-rational point on $X_{1}(N)$. Then there exists an elliptic curve $E / k$ with a $k$-rational point $P$ of order $N$ such that (the isomorphism class containing) the pair $(E, \pm P$ ) represents $x$ ([4] VI (3.2)). For $1 \neq \sigma \in \mathrm{Gal}(k / Q), x^{\sigma}$ is represented by the pair $\left(E^{\sigma}, \pm P^{\sigma}\right)$. For the integers $N, 1 \leqq N \leqq 10$ or $N=12, X_{1}(N) \simeq \boldsymbol{P}^{1}$. For $N=11,14$ and 15 , $X_{1}(N)$ are elliptic curves. For $N=13,16$ and 18, $X_{1}(N)$ are hyperelliptic curves of genus 2 . In this section, we prove the following theorem.

THEOREM (2.1). Let $N$ be an integer of a product of 2,3,5, 7, 11 and 13. If $X_{1}(N)$ is of genus $\geqq 2$ and is not hyperelliptic, then $Y_{1}(N)(k)=\phi$ for any quadratic field $k$.

Proof. It suffices to discuss the cases for the following integers $N=$ $2 \cdot 13,3 \cdot 13,5 \cdot 13,7 \cdot 13,11 \cdot 13 ; 2 \cdot 11,3 \cdot 11,5 \cdot 11,7 \cdot 11 ; 3 \cdot 7,4 \cdot 7,5 \cdot 7 ; 4 \cdot 5,6 \cdot 5$, $9 \cdot 5 ; 8 \cdot 3,4.9$ (see $[8,12][23])$. Suppose that there exists a $k$-rational point $x$ on $Y_{1}(N)$. Let $(E, \pm P) / k$ be a pair which represents $x$ with a $k$-rational point $P$ of order $N$ and let $1 \neq \sigma \in \operatorname{Gal}(k / Q)$. 
Case $N=13 q$ for $q=2,3,5,7$ and 11: We make use of the following lemma.

Lemma (2.2) ([23] (3.2)). Let $y$ be a k-rational point on $Y_{1}(13)$. Then the set $\{y,[5](y)\}$ represents a $\boldsymbol{Q}$-rational point on $X_{1}(13) /\langle[5]\rangle \simeq \boldsymbol{P}_{\boldsymbol{Q}}^{1}$, where [5] is the automorphism of $X_{1}(13)$ represented by $g \in \Gamma_{0}(13)$ such that $g \equiv$ $\left(\begin{array}{ll}5 & * \\ 0 & *\end{array}\right) \bmod 13$.

Let $\pi: X_{1}(13 q) \rightarrow X_{1}(13)$ be the natural morphism and $y$ be the $Q$ rational point $\{\pi(x),[5] \pi(x))\}$ on $Y_{1}(13) /\langle[5]\rangle$. Let $p$ be a prime of $k$ lying over the rational prime $p=3$ if $q=2$, and $p=5$ if $q \geqq 3$. Then the condition $Z / N Z \subset E(k)$ leads that $(Z / N Z)_{/ R} \subset E_{/ R}$, where $R$ is the localization $\left(\mathcal{O}_{k}\right)_{(p)}$ of $\mathcal{O}_{k}$ at $p(1.12)$. Then $E_{/ R}$ has multiplicative reduction cf. (1.12). Let $F$ be an elliptic curve defined over $\boldsymbol{Q}$ with a $\boldsymbol{Q}$-rational set $\{ \pm Q, \pm 5 Q\}$ for a point $Q$ of order 13 such that the pair $(F,\{ \pm Q, \pm 5 Q\})$ represents $y$ on $Y_{1}(13) /\langle[5]\rangle$. Let $\rho=\rho_{q}$ be the representation of the Galois action of $G=\operatorname{Gal}(\overline{\boldsymbol{Q}} / \boldsymbol{Q})$ on the $q$-torsion points $F_{q}(\overline{\boldsymbol{Q}})$. Then $F \simeq E$ over a quadratic extension $K$ of $k$, since $E$ has multiplicative reduction at $p$. Then for $G_{K}=\operatorname{Gal}(\overline{\boldsymbol{Q}} / K)$,

$$
\rho\left(G_{K}\right) \subset\left\{\left(\begin{array}{ll}
1 & * \\
0 & *
\end{array}\right)\right\} \subset \mathrm{GL}_{2}\left(\boldsymbol{F}_{q}\right) \simeq \operatorname{Aut} F_{q}(\overline{\boldsymbol{Q}}) .
$$

When $q=2, \mathrm{GL}_{2}\left(\boldsymbol{F}_{q}\right) \simeq \mathscr{S}_{3}$ (= the symmetric group of three letters) and

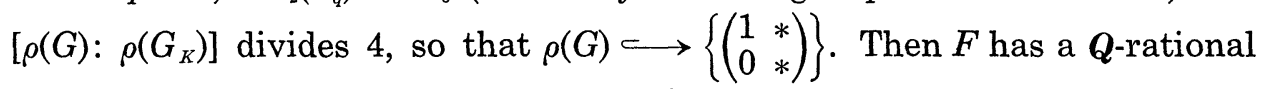
point $Q_{2}$ of order 2 and the pair $\left(F,\left\langle Q_{2}, Q\right\rangle\right)$ represents a $\boldsymbol{Q}$-rational point on $Y_{0}(26)$. But we know that $Y_{0}(26)(\boldsymbol{Q})=\phi([18][24]$ [36] table 1,5). Now consider the cases for $q \geqq 3$. Let $\theta_{q}$ be the cyclotomic character

$$
\theta_{q}: G=\operatorname{Gal}(\overline{\mathbf{Q}} / \boldsymbol{Q}) \longrightarrow \operatorname{Aut} \mu_{q}(\overline{\mathbf{Q}}) \text {. }
$$

Then det. $\rho=\theta_{q}$. Let $P_{q}$ be a $K$-rational point on $F$ of order $q$ and $g \in$ $G_{k} \backslash G_{K}$ for $G_{k}=\operatorname{Gal}(\overline{\mathbf{Q}} / k)$. If $P_{q}^{g} \neq \pm P_{q}$, then $\left\langle P_{q}^{g}\right\rangle \neq\left\langle P_{q}\right\rangle$ and $\rho\left(G_{K}\right)=$ $\{1\}$. Then $\theta_{q}\left(G_{K}\right)=\{1\}$, hence $q=3$, or $q=5$ and $K=Q\left(\zeta_{5}\right)$. For $q=3$, if $k \neq \boldsymbol{Q}\left(\zeta_{3}\right)$, then $K$ is an abelian extension of $\boldsymbol{Q}$ with the Galois group $\simeq Z / 2 Z \times Z / 2 Z$ and $\rho(G) \hookrightarrow\left\{\left(\begin{array}{ll}* & 0 \\ 0 & *\end{array}\right)\right\}$. If $k=\boldsymbol{Q}\left(\zeta_{3}\right)$, then $\rho\left(G_{k}\right)=\{ \pm 1\}$, since $\operatorname{det} \rho\left(G_{k}\right)=\theta_{3}\left(G_{k}\right)=\{1\}$. Then $\rho(G) \subset\left\{\left(\begin{array}{ll}* & 0 \\ 0 & *\end{array}\right)\right\}$, since $\theta_{3}(G)=\{ \pm 1\}$. For $q=5, K=\boldsymbol{Q}\left(\zeta_{5}\right)$ and $\rho(G) \longrightarrow\left\{\left(\begin{array}{ll}* & 0 \\ 0 & *\end{array}\right)\right\}$. Thus there exists a subgroup 
$A_{q} / \boldsymbol{Q}$ of $F$ of order $q$. Then the pair $\left(F, A_{q}+\langle Q\rangle\right)$ represents a $\boldsymbol{Q}$-rational point on $Y_{0}(13 q)$. But we know that $Y_{0}(13 q)(\boldsymbol{Q})=\phi$ for $q \geqq 2([9,10,11]$ [18] [20]). Now suppose that $P_{q}^{g}= \pm P_{q}$. Then $\rho\left(G_{k}\right) \longleftrightarrow\left\{\left(\begin{array}{ll} \pm 1 & * \\ 0 & *\end{array}\right)\right\}$. Take $h \in G \backslash G_{k}$ and put $A_{q}=\left\langle P_{q}\right\rangle$. If $A_{q}^{h}=A_{q}$, then the pair $\left(F, A_{q}+\langle Q\rangle\right)$ represents a $Q$-rational point on $Y_{0}(13 q)$. Therefore, $A_{q}^{h} \neq A_{q}$ and $\rho\left(G_{k}\right)$ $\hookrightarrow\left\{\left(\begin{array}{rr} \pm 1 & 0 \\ 0 & \pm 1\end{array}\right)\right\}$. If $\rho\left(G_{k}\right) \longleftrightarrow\left\{ \pm\left(\begin{array}{ll}1 & 0 \\ 0 & 1\end{array}\right)\right\}$, then $q=3, k=\boldsymbol{Q}\left(\zeta_{3}\right)$ and $\rho(G)$ $\longrightarrow\left\{ \pm\left(\begin{array}{ll}* & 0 \\ 0 & *\end{array}\right)\right\}$ and the same argument as above gives a contradiction. If $\rho\left(G_{k}\right) \simeq\left\{\left(\begin{array}{lr} \pm 1 & 0 \\ 0 & \pm 1\end{array}\right)\right\}$, then $q=3$ and $\rho(G)$ is contained in the normalizer of a split Cartan subgroup (, since $\operatorname{det} \rho=\theta_{q}$ ). Let $Y$ be the modular curve $/ \boldsymbol{Q}$ which corresponds to the modular group

$$
\left\{\left(\begin{array}{ll}
a & b \\
c & d
\end{array}\right) \in \Gamma_{0}(13) \mid b \equiv c \equiv 0 \text { or } a \equiv d \equiv 0 \bmod 3\right\} .
$$

Let $w$ be the involution of $Y$ represented by a matrix $g \in \Gamma_{0}(13)$ such that $g \equiv\left(\begin{array}{rr}0 & -1 \\ 1 & 0\end{array}\right) \bmod 3 . \quad$ Then the isomorphism of $X_{0}(9 \cdot 13)$ to $Y$ :

$$
\left(C, A_{9}+A_{13}\right) \longmapsto\left(C / A_{3},\left\{A_{9} / A_{3}, C_{3} / A_{3}\right\},\left(A_{13}+A_{3}\right) / A_{3}\right)
$$

induces an isomorphism of $X_{0}(9 \cdot 13) /\left\langle w_{9}\right\rangle$ to $Z=Y /\langle w\rangle$, where $A_{m}$ are cyclic subgroups of order $m$ with $A_{3} \subset A_{9}$. The jacobian variety $J=J(Z)$ of $Z$ has an optimal quotient $A / Q(J \longrightarrow A)$ with finite Mordell-Weil group ([36] table 1,5). As was seen as above, $F$ has potentially mutiplicative reduction at 5 . Let $z$ be the $Q$-rational point on $Y$ represented by $(F,\langle Q\rangle)$ with a level structure mod 3, then $z \otimes F_{5}=C \otimes F_{5}$ for a $\boldsymbol{Q}$-rational cusp $C$ on $Z$. Let $f: Z \rightarrow J \rightarrow A$ be the morphism defined by $f(y)=$ $c l((y)-(C))$. Then we see that $f(z)=0$ (see (1.11)). Let $\mathscr{Z}$ denote the normalization of $\mathscr{X}_{0}(1)$ in $Z$. Then we see that $f \otimes Z_{5}: \mathscr{Z} \otimes Z_{5} \rightarrow A_{/ Z_{5}}$ is a formal immersion along the cusp $C$ (see the proof in [22] (2.5)). Therefore, Mazur's method in [18] Section 4 can be applied to yield $z=C$. Thus we get a contradiction.

Case $N=11 q$ for $q=2,3,5$ and $7: q=2$ and 3: Let $p$ be a prime of $k$ lying over the rational prime 3 and put $R=\left(\mathcal{O}_{k}\right)_{(p)}$. The condition $Z / N Z \subset E(k)$ shows that $(Z / N Z)_{/ R} \subset E_{/ R}$ if $q=2$ or $q=3$ is unramified (1.11). If $q=3$ ramifies in $k$, then $(\boldsymbol{Z} / 11 \boldsymbol{Z})_{/ R} \subset E_{/ R}$ and $k(p)=\boldsymbol{F}_{3}$. Hence $x \otimes k(p)$ is also a cusp (see (1.12)). Denote also by $x, x^{\sigma}$ the images of $x$ and $x^{\sigma}$ under the natural morphism $\pi: X_{1}(N) \rightarrow X_{0}(N)$. Then $x \otimes \kappa(p)=$ 
$C \otimes \kappa(p), x^{\sigma} \otimes \kappa(p)=C_{\sigma} \otimes \kappa(p)$ for $Q$-rational cusps $C$ and $C_{\sigma}$ on $X_{0}(N)$. Let $i(x)=\operatorname{cl}\left((x)+\left(x^{\sigma}\right)-(C)-\left(C_{\sigma}\right)\right)$ be the $Q$-rational section of $J_{0}(N)_{/ Z}$. The Mordell-Weil groups of $J_{0}(11 q)$ for $q=2$ and 3 are finite and their orders are prime to 3 [36] table 1,3,5. Therefore $i(x)=0$, see (1.13). Since $Y_{0}(11 q)(\boldsymbol{Q})=\phi[18], C_{\sigma}=w_{22}(C)$ if $q=2$ and $C_{\sigma}=w_{11}(C)$ if $q=3$ (see (1.6)). As was seen as above, $C$ and $C_{\sigma}$ are represented by $\left(\boldsymbol{G}_{m} \times Z / 11 m Z, H\right)$ and $\left(\boldsymbol{G}_{m} \times Z / 11 m_{o} Z, H_{\sigma}\right)$ for integers $m, m_{\sigma} \geqq 1$ and cyclic subgroup $H, H_{\sigma}$ containing the subgroup $\simeq Z / 11 Z$. Thus we get a contradiction, since $w_{22}(C), w_{11}(C)$ are represented by $\left(\boldsymbol{G}_{m} \times \boldsymbol{Z} / m^{\prime} Z, H^{\prime}\right)$ for integers $m^{\prime}$ prime to 11 [4] VII.

$q=5:$ Let $X$ be the subcovering as in (1.3):

$$
X_{1}(55) \stackrel{\pi_{1}}{\longrightarrow} X \stackrel{\pi_{X}}{\longrightarrow} X_{0}(55) \text {. }
$$

Let $1 \neq \gamma \in \operatorname{Gal}\left(X / X_{0}(55)\right)$ and $\delta$ be the automorphism of $X$ defined by

$$
\left(F, \pm P_{5}, B_{11}\right) \longmapsto\left(F / B_{11}, \pm 2 P_{5} \bmod B_{11}, E_{11} / B_{11}\right)
$$

where $P_{5}$ is a point of order 5 and $B_{11}$ is a subgroup of order 11 . Then $\delta$ has 16 fixed points (1.8). Let $p$ be a prime of $k$ lying over the rational prime 5 and put $R=\left(\mathcal{O}_{k}\right)_{(p)}$. The condition $Z / 55 Z \subset E(k)$ shows that $x \otimes \kappa(p)=C \otimes \kappa(p), x^{\sigma} \otimes \kappa(p)=C_{\sigma} \otimes \kappa(p)$ for 0-cusps $C$ and $C_{\sigma}$ (see (1.11), (1.12)). Denote also by $x, x^{\sigma}, C$ and $C_{\sigma}$ the images of $x, x^{\sigma}, C$ and $C_{\sigma}$ under the natural morphism $\pi_{1}: X_{1}(55) \rightarrow X$. Put $C_{X}=\operatorname{Coker}\left(\pi_{X}^{*}: J_{0}(55) \rightarrow J(X)\right)$, which has the Mordell-Weil group of finite order (1.5). Let $i(x)=\operatorname{cl}((x)$ $\left.+\left(x^{\sigma}\right)-(C)-\left(C_{\sigma}\right)\right)$ be the $\boldsymbol{Q}$-rational section of $J(X)_{/ Z}$. Then $i(x) \otimes F_{5}=0$ (1.13), so by (1.11), $i(x) \in \pi_{X}^{*}\left(J_{0}(55)\right)$. Then we get a rational function $f$ on $X$ such that

$$
(f)=(x)+\left(x^{\sigma}\right)+(\gamma(C))+\left(\gamma\left(C_{\sigma}\right)\right)-(\gamma(x))-\left(\gamma\left(x^{\sigma}\right)\right)-(C)-\left(C_{\sigma}\right) .
$$

Since $\gamma(C) \otimes F_{5} \neq C \otimes F_{5}, \gamma(x) \neq x$. If $f$ is a constant function, then $\gamma(x)$ $=x^{\sigma}$ and the set $\left\{x, \gamma(x)=x^{\sigma}\right\}$ defines a $Q$-rational point on $Y_{0}(55)$. But $Y_{0}(55)(\boldsymbol{Q})=\phi[18]$, so that $f$ is not a constant function. If $\left(\delta^{*} f\right)=(f)$, then $\delta(C)=C$ or $C_{o}$. But $C, C_{o}$ are 0 -cusps and $\delta(C)$ is not a 0-cusps, so that $\left(\delta^{*} f\right) \neq(f)$. Applying (1.9) to $f$ and $\delta$, we get a contradiction.

Remark (2.3). For any cubic field $k^{\prime}, Y_{1}(55)\left(k^{\prime}\right)=\phi$. It is shown by the same way as above, taking a prime $p^{\prime} \mid 5$ of the smallest Galois extension of $\boldsymbol{Q}$ containing $k^{\prime}$. 
$q=7:$ Let $\pi_{11}: X_{0}(77) \rightarrow X_{0}(77) /\left\langle w_{11}\right\rangle$ be the natural morphism and $J^{\prime}$ be the jacobian variety of $X_{0}(77) \mid\left\langle w_{11}\right\rangle$. Then $A=\operatorname{Coker}\left(\pi_{11}^{*}: J^{\prime} \rightarrow J_{0}(77)\right)$ has the Mordell-Weil group of finite order [36] table 1,5. Let $p$ be a prime of $k$ lying over the rational prime 5 . The condition $Z / 77 Z \subset E(k)$ shows that $x \otimes \kappa(p)$ is a 0 -cusp $(\otimes \kappa(p))(1.12)$. Denote also by $x, x^{\sigma}$ the images of $x$ and $x^{\sigma}$ under the natural morphism $X_{1}(77) \rightarrow X_{0}(77)$. Then $x \otimes \kappa(p)=$ $\mathbf{0} \otimes \kappa(p)$. Let $i(x)=c l\left((x)+\left(x^{\sigma}\right)-2(0)\right)$ be the $\boldsymbol{Q}$-rational section of $J_{0}(77)_{/ Z}$. Then $i(x) \otimes \boldsymbol{F}_{5}=0$ and $i(x) \in \pi_{11}^{*}\left(J^{\prime}\right)$ (see (1.11), (1.13)). Then we get a rational function $f / Q$ on $X_{0}(77)$ such that

$$
(f)=(x)+\left(x^{\sigma}\right)+2\left(w_{11}(0)\right)-\left(w_{11}(x)\right)-\left(w_{11}\left(x^{\sigma}\right)\right)-2(0) .
$$

Then $\left(w_{11}^{*} f\right)=-(f) \neq 0$, since $w_{11}(\mathbf{0}) \neq \mathbf{0}$. Hence $w_{11}^{*} f=\alpha / f$ for $\alpha \in \boldsymbol{Q}^{\times}$. The fundamental involution $w=w_{77}$ of $X_{0}(77)$ has 8 fixed points $x_{i}(1 \leqq i \leqq 8)$. The cusps $w_{11}(\mathbf{0}) \otimes \boldsymbol{F}_{5}$ and $\mathbf{0} \otimes \boldsymbol{F}_{5}$ are not the fixed point of $w$. Therefore by (1.9),

$$
\left(w^{*} f \mid f-1\right)_{0}=\sum_{i=1}^{8}\left(x_{i}\right)(\underset{\mathrm{put}}{=} D) .
$$

Put $g=\left(w^{*} f / f-1\right)^{-1}$. Then

$$
(g)=(x)+\left(x^{\sigma}\right)+2\left(w_{11}(0)\right)+\left(w_{7}(x)\right)+\left(w_{7}\left(x^{\sigma}\right)\right)+2(\infty)-D
$$

and

$$
w^{*} g=w_{11}^{*} g=-1-g .
$$

Then $g$ defines a rational function $h$ on $Y=X_{0}(77) /\left\langle w_{7}\right\rangle$ with $\pi_{7}^{*}(h)=g$, where $\pi_{7}: X_{0}(77) \rightarrow Y$ is the natural morphism. Set $\left\{y_{i}\right\}_{1 \leqq i \leqq 4}=\left\{\pi_{7}\left(x_{j}\right)\right\}$, and put $E=\sum_{i=1}^{4}\left(y_{i}\right)$ and $C=\pi_{7}(\infty)\left(=\pi_{7}\left(w_{7}(0)\right)\right)$. Then $h$ is of degree 4 and $h \in H^{\circ}\left(Y, \mathcal{O}_{Y}(E-2(C))\right)$. Denote also by $w$ the involution of $Y$ induced by $w\left(\right.$ and $\left.w_{11}\right)$. Then

$$
w^{*} h=-1-h \text { and }(h)_{\infty}=E .
$$

Let $\pi_{Y}: Y \rightarrow Z=X_{0}(77) /\left\langle w_{7}, w_{11}\right\rangle$ be the natural morphism. $Z$ is an elliptic curve [36] table 5. The canonical divisor $K_{Y} \sim E$ (linearly equivalent) and $\operatorname{dim} H^{0}\left(Y, \mathcal{O}_{Y}(E)\right)=3$. Let $\omega$ be the base of $H^{\circ}\left(Z, \Omega^{1}\right)$ and $\omega_{1}=\pi_{Y}^{*}(\omega), \omega_{2}$ and $\omega_{3}$ be the basis of $H^{\circ}\left(Y, \Omega^{1}\right)$ such that $\omega_{i}(C)=1$ and that $\omega_{i}$ are eigen forms of the Hecke ring $Q\left[T_{m}, w\right]_{(m, 77)=1}$ with $T_{2}^{*} \omega_{2}=0$ and $T_{2}^{*} \omega_{3}=\omega_{3}$ (see [36] table 1,3,5). Then $\left\{1, f_{2}=\omega_{2} / \omega_{1}, f_{3}=\omega_{3} / \omega_{1}\right\}$ is the set of basis of $H^{0}\left(Y, \mathcal{O}_{Y}(E)\right)$ such that $f_{2}=1+q+\cdots$ and $f_{3}=1-3 q+\cdots$ for $q=$ $\exp (2 \pi \sqrt{-1} z)$ (see loc. cit.). Then $h=a_{1}+a_{2} f_{2}+a_{3} f_{3}$ for $a_{i} \in \boldsymbol{Q}$. The 
conditions $w^{*} h=-1-h$ and $w^{*} f_{i}=-f_{i}$ show that $a_{1}=-\frac{1}{2}$. Further by the condition $(h)_{0} \succ 2(C), a_{2}=\frac{1}{3}$ and $a_{3}=\frac{1}{6}$. Let $\mathscr{Y}$ be the quotient $\mathscr{X}_{0}(77) /\left\langle w_{7}\right\rangle \otimes Z_{5}$ and $\widehat{\mathcal{O}_{\mathscr{Y}}, C}$ be the completion of the local ring $\mathcal{O}_{\mathscr{Y}, C}$ along the cuspidal section $C$. Then $f_{i} \in \widehat{\mathcal{O}_{\mathscr{y}}, C}$, so that $h \in \widehat{\mathcal{O}_{y, C}}$. Put $C^{\prime}=\pi_{7}(\mathbf{0})(=$ $\left.\pi_{7}\left(w_{7}(0)\right)\right)$. Then $w^{*} h \in \widehat{\mathcal{O}_{\mathscr{y}, C^{\prime}}}$ and $w^{*} h\left(\pi_{Y}(x)\right)=(-1-h)\left(\pi_{Y}(x)\right)=-1, w^{*} h\left(C^{\prime}\right)$ $=(-1-g)(0)=0$. But the conditions that $x \otimes \kappa(p)=\mathbf{0} \otimes \kappa(p)$ for $p(\mid 5)$ and $w^{*} h \in \widehat{\mathcal{O}_{\mathscr{y}, C^{\prime}}}$ give the congruence $w^{*} h\left(\pi_{Y}(x)\right) \equiv w^{*} h\left(C^{\prime}\right) \bmod p$. Thus we get a contradiction.

Case $N=7 n$ for $n=3,4$ and 7 :

$n=3$ : Let $X$ be the subcovering as $n(1.3)$ :

$$
X_{1}(21) \stackrel{2}{\longrightarrow} X \stackrel{3}{\longrightarrow} X_{0}(21),
$$

which corresponds to the subgroup $A=(Z / 3 Z)^{\times} \times\{ \pm 1\}$. Let $\mathscr{X}$ denote the normalization of $\mathscr{X}_{0}(1)$ in $X$. The special fibre $\mathscr{X} \otimes F_{3}$ is reduced (1.2). Let $p$ be a prime of $k$ lying over the rational prime 3 and put $R=\left(\mathcal{O}_{k}\right)_{(p)}$. The condition $Z / 21 Z \subset E(k)$ shows that $(Z / 21 Z)_{/ R} \subset E_{/ R}$ if the rational prime 3 is unramified in $k(1.11)$, (1.12). If 3 ramifies in $k$, then $k(p)=\boldsymbol{F}_{3}$, so that in both cases $E_{/ R}$ has multiplicative reduction see (1.12). Therefore, $x \otimes \kappa(p)=C \otimes \kappa(p), x^{\sigma} \otimes \kappa(p)=C_{\sigma} \otimes \kappa(p)$ for $\boldsymbol{Q}$-rational cusps $C$ and $C_{\sigma}$ (see loc. cit.). Let $i(x)=\operatorname{cl}\left((x)+\left(x^{\sigma}\right)-(C)-\left(C_{\sigma}\right)\right.$ ) be the $\boldsymbol{Q}$-rational section of $J(X)_{/ Z}$. Since the Mordell-Weil group of $J(X)$ is finite (1.4), (1.5), $(x)+\left(x^{\sigma}\right) \sim(C)+\left(C_{\sigma}\right)$. But $X$ is not hyperelliptic (1.7).

$n=4$ : Let $p$ be a prime of $k$ lying over the rational prime 3 and put $R=\left(\mathcal{O}_{k}\right)_{(p)}$. The condition $Z / 28 Z \subset E(k)$ shows that $(Z / 28 Z)_{/ R} \subset E_{/ R}$. Denote also by $x, x^{\sigma}$ the images of $x$ and $x^{\sigma}$ under the natural morphism $X_{1}(28) \rightarrow X_{0}(28)$. Then $x \otimes \kappa(p)=C \otimes \kappa(p), x^{\sigma} \otimes \kappa(p)=C_{\sigma} \otimes \kappa(p)$ for $\boldsymbol{Q}$ rational cusps $C$ and $C_{\sigma}$. These cusps $C, C_{\sigma}$ are represented by $\left(\boldsymbol{G}_{m} \times \boldsymbol{Z} / 7 m \boldsymbol{Z}, \boldsymbol{H}\right)$ and $\left(\boldsymbol{G}_{m} \times \boldsymbol{Z} / 7 m_{\sigma} \boldsymbol{Z}, H_{\sigma}\right)$ for integers $m$ and $m_{\sigma}$ and cyclic subgroups $H, H_{\sigma}$ containing $\{1\} \times m Z / 7 m Z$ and $\{1\} \times m_{\sigma} Z / 7 m_{\sigma} Z$, respectively. Let $i(x)=\operatorname{cl}\left((x)+\left(x^{\sigma}\right)-(C)-\left(C_{\sigma}\right)\right)$ be the $Q$-rational section of $J_{0}(28)_{/ Z}$. Since the Mordell-Weil group of $J_{0}(28)$ is finite (1.4), $i(x)=0$ (1.13) and $(x)+\left(x^{\sigma}\right) \sim(C)+\left(C_{\sigma}\right) . \quad X_{0}(28)$ has the hyperelliptic involution $w_{7}$, so $C_{\sigma}$ $=w_{7}(C)$. But as noted as above, $C_{\sigma} \neq w_{7}(C)$.

$n=5$ : Let $X$ be the subcovering as in (1.3):

$$
X_{1}(35) \stackrel{\pi_{1}}{\longrightarrow} X \stackrel{\pi_{X}}{\longrightarrow} X_{0}(35) \text {, }
$$


which corresponds to the subgroup $\Delta=(Z / 5 Z)^{\times} \times\{ \pm 1\}$. The automorphism $\gamma$ of $X$ represented by

$$
\left(F, B_{5}, \pm Q_{7}\right) \longmapsto\left(F / B_{5}, F_{5} / B_{5}, \pm 3 Q_{7} \bmod B_{5}\right)
$$

has 12 fixed points (1.8). Let $p$ be a prime of $k$ lying over the rational prime 3 and put $R=\left(\mathcal{O}_{k}\right)_{(p)}$. The condition $Z / 35 Z \subset E(k)$ shows that $(Z / 35 Z)_{/ R} \subset E_{/ R}$. Denote also by $x, x^{\sigma}$ the images of $x$ and $x^{\sigma}$ by the natural morphism $\pi_{1}: X_{1}(35) \rightarrow X$. Then $x \otimes \kappa(p)=C \otimes \kappa(p), x^{\sigma} \otimes \kappa(p)=C_{\sigma} \otimes \kappa(p)$ for $\boldsymbol{Q}$-rational cusps $C$ and $C_{\sigma}(1.12)$. Let $i(x)=\operatorname{cl}\left((x)+\left(x^{\sigma}\right)-(C)-\left(C_{\sigma}\right)\right)$ be the $Q$-rational section of $J(X)_{/ Z}$. The Mordell-Weil group of $C_{X}=$ $\operatorname{Coker}\left(\pi_{X}^{*}: J_{0}(35) \rightarrow J(X)\right)$ is finite (1.5). Let $\delta$ be a generator of $\mathrm{Gal}\left(X / X_{0}(35)\right)$. Then we get a rational function $f$ on $X$ such that

$$
(f)=(x)+\left(x^{\sigma}\right)+(\delta(C))+\left(\delta\left(C_{o}\right)\right)-(\delta(x))-\left(\delta\left(x^{\sigma}\right)\right)-(C)-\left(C_{\sigma}\right)
$$

(see (1.13)). If $f$ is a constant function, then $\left\{x, x^{\sigma}\right\}=\left\{\delta(x), \delta\left(x^{\sigma}\right)\right\}$. Then $x=\delta(x)=\delta^{2}(x)$, hence $C \otimes \kappa(p)=\delta(C \otimes \kappa(p))$. But $C \otimes \kappa(p)$ is not a fixed point of $\delta$. The similar argument as above shows that $\left(\gamma^{*} f\right) \neq(f)$. Applying (1.9) to $f$ and $\gamma$, we get a contradiction.

Case $N=5 n$ for $n=4,6$ and 9 :

$n=4$ : Let $p$ be a prime of $k$ lying over the rational prime 3 and put $R=\left(\mathcal{O}_{k}\right)_{(p)}$. The condition $Z / 20 Z \subset E(k)$ shows that $(Z / 20 Z)_{/ R} \subset E_{/ R}$ and that $E_{/ R}$ has multiplicative reduction (1.12). Let $T$ be the connected component of the special fibre $E_{/ R} \otimes \kappa(p)$ of the unit section. If $p$ is of degree one, then $Z / 5 Z \not \subset T\left(F_{3}\right)$. Then $x \otimes \kappa(p)=C \otimes \kappa(p), x^{\sigma} \otimes \kappa(p)=$ $C_{\sigma} \otimes \kappa(p)$ for $Q$-rational cusps $C$ and $C_{\sigma}$, since $\left(\frac{-1}{3}\right)=-1$, where $\left(\frac{-1}{}\right)$ is the quadratic residue symbol. If $p$ is of degree two, then $x \otimes \kappa(p)=$ $C \otimes \kappa(p)$ for a $Q(\sqrt{-1})$-rational cusp $C$, and $x^{\sigma} \otimes \kappa(p)=C_{\sigma} \otimes \kappa(p)$ with $C_{\sigma}=C^{\tau}$ for $1 \neq \tau \in \operatorname{Gal}(\boldsymbol{Q}(\sqrt{-1}) / Q)$. Let $i(x)=\operatorname{cl}\left((x)+\left(x^{\sigma}\right)-(C)-\left(C_{\sigma}\right)\right)$ be the $Q$-rational section of $J_{1}(20)_{/ Z}$. Since $\sharp J_{1}(20)(\boldsymbol{Q})<\infty$ (1.4) (1.5), $i(x)$ $=0$ (1.14) and $(x)+\left(x^{\sigma}\right) \sim(C)+\left(C_{\sigma}\right)$. But $X_{1}(20)$ is not hyperelliptic (1.7).

$n=6$ : The modular curve $X_{0}(30)$ has the hyperelliptic involution $w_{15}$ : $(F, B) \mapsto\left(F / B_{15},\left(B+F_{15}\right) / B_{15}\right)$, where $B_{15}$ is the subgroup of $B$ of order 15 . Let $p$ be a prime of $k$ lying over the rational prime 3 and put $R=\left(\mathcal{O}_{k}\right)_{(p)}$. Then $(Z / 10 Z)_{/ R} \subset E_{/ R}$ and $E_{/ R}$ is semistable (1.12). If 3 is unramified in $k$, then $(Z / 30 Z)_{/ R} \subset E_{/ R}$. Then $E_{/ R}$ has multiplicative reduction and $(Z / 3 Z)_{/ R} \otimes \kappa(p)$ is not contained in the connected component of thə special 
$E_{/ R} \otimes \kappa(p)$ of the unit section (see (1.11), (1.12)). If 3 ramifies in $k$, then $E_{/ R}$ has also mutliplicative reduction and $(Z / 5 Z)_{/ R} \otimes k(p)$ is not containted in the connected component of $E_{/ R} \otimes k(p)$ of the unit section (see loc. cit.). Denote also by $x, x^{\sigma}$ the images of $x$ and $x^{\sigma}$ under the natural morphism $X_{1}(30) \rightarrow X_{0}(30)$. Then $x \otimes \kappa(p)=C \otimes \kappa(p), x^{\sigma} \otimes \kappa(p)=C_{\sigma} \otimes \kappa(p)$ for $\mathbf{Q}$ fibre rational cusps $C$ and $C_{\sigma}$. These cusps $C, C_{\sigma}$ are represented by $\left(\boldsymbol{G}_{m} \times \boldsymbol{Z} / q m_{o} \boldsymbol{Z}, \boldsymbol{H}_{\sigma}\right)$ and $\left(\boldsymbol{G}_{m} \times \boldsymbol{Z} / q m_{o} \boldsymbol{Z}, H_{o}\right)$ for integers $m, m_{\sigma} \geqq 1$ and cyclic subgroups $H, H_{\sigma}$ containing $\{1\} \times m Z / q m Z$ and $\{1\} \times m_{\sigma} Z / q m_{\sigma} Z$ for $q=3$ or 5 , respectively. Let $i(x)=\operatorname{cl}\left((x)+\left(x^{o}\right)-(C)-\left(C_{o}\right)\right)$ be the $\boldsymbol{Q}$-rational section of $J_{0}(30)_{/ Z}$. Since $\# J_{0}(30)(Q)<\infty(1.4), i(x)=0(1.13)$ and $(x)+\left(x^{o}\right)$ $\sim(C)+\left(C_{\sigma}\right)$. It yields $w_{15}(C)=C_{\sigma}$. But as noted as above, $w_{15}(C) \neq C_{\sigma}$.

$n=9$ : Let $p$ be a prime of $k$ lying over the rational prime 5 and put $R=\left(\mathcal{O}_{k}\right)_{(p)}$. Then $(Z / 45 Z)_{/ R} \subset E_{/ R}$ and $x \otimes \kappa(p)=C \otimes \kappa(p), x^{\sigma} \otimes \kappa(p)=C_{\sigma}$ $\otimes \kappa(p)$ for 0 -cusps $C$ and $C_{\sigma}$ (1.11), (1.12). Denote also by $x, x^{\sigma}, C$ and $C_{\sigma}$ the images of $x, x^{\sigma}, C$ and $C_{o}$ under the natural morphism $X_{1}(45) \rightarrow X_{0}(45)$. Let $i(x)=\operatorname{cl}\left((x)+\left(x^{o}\right)-(C)-\left(C_{\sigma}\right)\right)$ be the $\boldsymbol{Q}$-rational section of $J_{0}(45)_{/ Z}$. Since $\left.\# J_{0}(45)_{/ Z}\right)(\boldsymbol{Q})<\infty(1.4), i(x)=0$ (1.13). But $X_{0}(45)$ is not hyperelliptic [25].

Case $N=3 n$ for $n=8$ and 12 :

$n=8$ : Let $X$ be the subcovering as in (1.3):

$$
X_{1}(24) \stackrel{\pi_{1}}{\longrightarrow} X \stackrel{\pi_{X}}{\longrightarrow} X_{0}(24) \text {, }
$$

which corresponds to the subgroup $\Delta=\{ \pm 1\} \times(\boldsymbol{Z} / 3 \boldsymbol{Z})^{\times}$. Let $p$ be a prime of $k$ lying over the rational prime 3 and put $R=\left(\mathcal{O}_{k}\right)_{(p)}$. Then $(Z / 8 Z)_{/ R} \subset E_{/ R}$ and $E_{/ R}$ is semistable (1.12). If 3 is unramified in $k$, then $(Z / 24 Z)_{/ R} \subset E_{/ R}$ (1.11) and $E_{/ R}$ has multiplicative reduction (1.12). If 3 ramifies in $k$, then $p$ is of degree one, so $E_{/ R}$ has also multiplicative reduction (see loc. cit.). Denote also by $x, x^{\sigma}$ the images of $x$ and $x^{\sigma}$ by the natural morphism $\pi: X_{1}(24) \rightarrow X$. If $p$ is of degree one, then $x \otimes k(p)$ $=C \otimes \kappa(p), x^{\sigma} \otimes \kappa(p)=C_{\sigma} \otimes \kappa(p)$ for $\boldsymbol{Q}$-rational cusps $C$ and $C_{\sigma}$. Any cusp on $X$ is defined over $\boldsymbol{Q}$ or $\boldsymbol{Q}(\sqrt{2})$. If $p$ is of degree two, then $x \otimes \kappa(p)$ $=C \otimes \kappa(p)$ for a $\boldsymbol{Q}(\sqrt{2})$-rational cusp $C$. Then $x^{\sigma} \otimes \kappa(p)=C_{\sigma} \otimes \kappa(p)$ for $C_{\sigma}=C^{r}$ and $\left.1 \neq \tau \in \operatorname{Gal}(Q \sqrt{2}) / Q\right)$, since $\left(\frac{2}{3}\right)=-1$. Let $i(x)=\operatorname{cl}((x)+$ $\left.\left(x^{\sigma}\right)-(C)-\left(C_{o}\right)\right)$ be the $\boldsymbol{Q}$-rational section of $J(X)_{/ \mathbf{Z}}$. Since $\sharp J(X)(\boldsymbol{Q})<$ $\infty$ (1.4) (1.5), $i(x)=0$ (1.13). But $X$ is not hyperelliptic (1.7).

$n=12$ : Let $p$ be a prime of $k$ lying over the rational prime 5 and put 
$R=\left(\mathcal{O}_{k}\right)_{(p)}$. Then $(Z / 36 Z)_{/ R} \subset E_{/ R}$ and $E_{/ R}$ is semistable (1.12). If $E_{/ R}$ has good reduction, then $\sharp E_{/ R}\left(F_{25}\right)=1+25-(-10)$ (, since $Z / 36 Z \subset E_{/ R}\left(F_{25}\right)$ and $\left.\sharp E_{/ R}\left(\boldsymbol{F}_{25}\right) \leqq 36\right)$. But then the Frobenius map $F=F_{25}: E_{/ R} \otimes \boldsymbol{F}_{25} \rightarrow$ $E_{/ R} \otimes F_{25}$ does not act trivially on $E_{/ R}\left(\boldsymbol{F}_{25}\right) \longleftrightarrow Z / 36 \boldsymbol{Z}$. Hence $E_{/ R}$ has multiplicative reduction. Let $T$ be the connected component of $E_{/ R} \otimes \kappa(p)$ of the unit section. Then $Z / 9 Z \not \subset T\left(F_{25}\right)$. Denote also by $x, x^{\sigma}$ the images of $x$ and $x^{\sigma}$ under the natural morphism $X_{1}(36) \rightarrow X_{1}(18)$. Then $x \otimes \kappa(p)$ $=C \otimes \kappa(p), x^{\sigma} \otimes \kappa(p)=C_{\sigma} \otimes \kappa(p)$ for $Q$-rational cusps $C$ and $C_{\sigma}$ on $X_{1}(18)$ (see above). The modular curve $X_{1}(18)$ has the hyperelliptic involution $w_{2}[5](1.6)$ :

$$
\left(F, B_{2}, \pm Q_{9}\right) \longmapsto\left(F / B_{2}, F_{2} / B_{2}, \pm 5 Q_{9} \bmod B_{2}\right),
$$

where $B_{2}$ is a subgroup of order 2 and $Q_{9}$ is a point of order 9 . Let $i(x)$ $=\operatorname{cl}\left((x)+\left(x^{\sigma}\right)-(C)-\left(C_{\sigma}\right)\right)$ be the $\boldsymbol{Q}$-rational section of $J_{1}(18)_{/ Z}$. Since $\sharp J_{1}(18)(\boldsymbol{Q})<\infty(1.4), i(x)=0$ (1.13) and $x^{\sigma}=w_{2}[5](x)$. For a $k$-rational point $Q \in\langle P\rangle$ of order 18 , the pairs $(E, \pm Q),\left(E^{\sigma}, \pm Q^{\sigma}\right)$ represent $x$ and $x^{\sigma}$ on $X_{1}(18)$. Put $A_{2}=\langle 9 Q\rangle$. Then there is a quadratic extension $K$ of $k$ over which

$$
\lambda:\left(E^{\sigma}, \pm Q^{\sigma}\right) \stackrel{\sim}{\longrightarrow}\left(E / A_{2}, \pm\left(Q_{2}^{\prime}+5 Q\right) \bmod A_{2}\right),
$$

where $Q_{2}^{\prime}$ is a point of order 2 not contained in $A_{2}$. For $1 \neq \tau \in \operatorname{Gal}(K / k)$, $\lambda^{\tau}= \pm \lambda$, since $x \otimes \kappa(p)$ is a cusp. Then $\lambda\left(Q^{\sigma}\right)=\varepsilon\left(Q_{2}^{\prime}+5 Q\right) \bmod \mathrm{A}_{2}$ for $\varepsilon= \pm 1$. The points $Q^{\sigma}$ and $\lambda\left(Q^{\sigma}\right)$ are $k$-rational, so $\lambda^{\tau}\left(Q^{\sigma}\right)=\left(\lambda\left(Q^{\sigma \tau}\right)\right)^{\tau}=$ $\lambda\left(Q^{\sigma}\right)$. Therefore $\lambda^{\tau}=\lambda$ and $\lambda$ is defined over $k$. Since $E / A_{2}$ contains $E_{2} / A_{2} \oplus\langle 9 P\rangle / A_{2}(\simeq Z / 2 Z \times Z / 2 Z), E^{\circ}(k) \supset Z / 2 Z \times Z / 36 Z$. Let $X_{0}(2,36)$ be the modular curve $/ Q$ corresponding to $\Gamma_{0}(2,36)$. Then $E$ and $E^{\sigma}$ (with level structures) define $k$-rational points $y$ and $y^{\sigma}$ on $X_{0}(2,36)$ such that $y \otimes \kappa(p)=D \otimes \kappa(p), y^{\sigma} \otimes \kappa(p)=D_{\sigma} \otimes \kappa(p)$ for $Q$-rational cusps $D$ and $D_{\sigma}$. Let $i(y)=\operatorname{cl}\left((y)+\left(y^{\sigma}\right)-(D)-\left(D_{\sigma}\right)\right)$ be the $\boldsymbol{Q}$-rational section of $J_{0}(2,36)_{/ Z}$. Then $i(y)=0$, since $\# J_{0}(2,36)(\boldsymbol{Q})<\infty(1.4)$ (1.13). But $X_{0}(2,36)$ is not hyperelliptic [25].

Now we discuss the $k$-rational points on $X_{1}(N)$ for $N=14,15$ and 18 . The modular curves $X_{1}(14)$ and $X_{1}(15)$ are elliptic curves, and $X_{1}(18)$ is hyperelliptic of genus 2 . We here give examples of quadratic fields $k$ such that $Y_{1}(N)(k)=\phi$ for each integer $N$ as above.

Proposition (2.4). Let $k$ be a quadratic field. If one of the following conditions (i), (ii) and (iii) is satisfied, then $Y_{1}(18)(k)=\phi$ : 
(i) The rational prime 3 remains prime in $k$.

(ii) 3 splits in $k$ and 2 does not split in $k$.

(iii) 5 or 7 ramifies in $k$.

Proof. Let $x$ be a $k$-rational point on $Y_{1}(18)$. Then $x$ is represented by an elliptic curve $E$ defined over $k$ with a $k$-rational point $P$ of order 18 [4] VI (32.). Let $p=2,3,5$ or 7 , and put $R=\left(\mathcal{O}_{k}\right)_{(p)}$ for a prime $p$ of $k$ lying over $p$. Then $(Z / 18 Z)_{/ R} \subset E_{/ R}$ if $p=5$ or $7,(Z / 9 Z)_{/ R} \subset E_{/ R}$ if $p=2$ and $(Z / 18 Z)_{/ R} \subset E_{/ R}$ if $p=3$ is unramified in $k$ (1.11).

Case (i) and (ii): The same argument as in the proof for $N=36$ shows that $x \otimes \kappa(p)=C \otimes \kappa(p), x^{\sigma} \otimes \kappa(p)=C_{\sigma} \otimes \kappa(p)$ for $Q$-rational cusps $C$ and $C_{\sigma}$ and for a prime $p$ of $k$ lying over $p=3$. Using the $Q$-rational section $i(x)=\operatorname{cl}\left((x)+\left(x^{\sigma}\right)-(C)-\left(C_{o}\right)\right)$ of $J_{1}(18)_{/ Z}$, we see that $w_{2}[5](C)$ $=C_{\sigma}$. If 3 remains prime in $k$, then $C_{\sigma} \otimes F_{9}=x^{\sigma} \otimes F_{9}=\left(x \otimes F_{9}\right)^{(3)}=C \otimes F_{9}$. But $C \otimes \boldsymbol{F}_{9}$ is not a fixed point of the hyperelliptic involution $w_{2}[5]$. In the case (ii), the same argument as above shows that $C \otimes F_{4}=C_{\sigma} \otimes F_{4}$. But $C \otimes F_{4}$ is not a fixed point of $w_{2}[5]$.

Case (iii): Under the assumption that $p=5$ or 7 ramifies in $k$, the same argument as above gives the result.

ExAmple (2.5). (1) $Y_{1}(14)(k)=\phi$ for $k=\boldsymbol{Q}(\sqrt{-3})$ and $\boldsymbol{Q}(\sqrt{-7})$.

(2) $Y_{1}(15)(\boldsymbol{Q}(\sqrt{5}))=\phi$.

Proof. For $N=14$ and $15, X_{0}(N)$ are elliptic curves with finite Mordell-Weil groups [36] table 1. The restriction of scalars [5] [34] $\operatorname{Re}_{\boldsymbol{Q}(\sqrt{-3}) / \boldsymbol{Q}}\left(X^{0}(14)_{/ \boldsymbol{Q}(\sqrt{-3})}\right), \operatorname{Re}_{/ \boldsymbol{Q}(\sqrt{-7}) / \boldsymbol{Q}}\left(X_{0}(14)_{/ \boldsymbol{Q}(\sqrt{-7})}\right)$ and $\operatorname{Re}_{\boldsymbol{Q}(\sqrt{5}) / \boldsymbol{Q}}\left(X_{0}(15)_{/ \boldsymbol{Q}(\sqrt{5})}\right)$ are isogenous over $\boldsymbol{Q}$ (respectively) to products $X_{0}(14) \times E_{126}, X_{0}(14) \times E_{98}$ and $X_{0}(15) \times E_{75}$ for elliptic curves $E_{n}$ with conductor $n$ (1.15). These $E_{n}$ have the Mordell-Weil groups of finite order [36] table 1. Therefore $\# X_{0}(N)(k)$ $<\infty$ for $(N, k)$ as above. Let $x$ be a $k$-rational point on $X_{1}(N)$ and denote also by $x$ the image of $x$ under natural morphism $X_{1}(N) \rightarrow X_{0}(N)$ for $(N, k)$ as above. Then $x \otimes \kappa(p)=C \otimes \kappa(p)$ for a $\boldsymbol{Q}$-rational cusp $C$ on $X_{0}(N)$ and for a prime $p$ of $k$ lying over $p=7$ if $N=14$, and $p=5$ if $N=15$ (1.11) (1.12). Then the specialization Lemma (1.11) yields that $x=C$.

\section{§3. Rational points on $X_{1}(m, N)$}

Let $N$ be an integer of a product of powers of $2,3,5,7,11$ and 13, and $m \neq 1$ be a positive divisor of $N$. Let $k$ be a quadratic field. In this 
section, we discuss the $k$-rational points on $X_{1}(m, N)$. For $(m, N)=(2,2)$, $(2,4),(2,6),(2,8) ;(3,3),(3,6) ;(4,4), X_{1}(m, N) \simeq \boldsymbol{P}^{1}$. For $(m, N)=(2,10)$ and $(2,12), X_{1}(m, N)$ are elliptic curves. For the other pairs $(m, N)$ as above, $X_{1}(m, N)$ are not hyperelliptic [7]. We first discuss the $k$-rational points on $Y_{1}(m, N)$ for the pairs $(m, N)$ such that $X_{1}(m, N)$ are not hyperelliptic. It suffices to treat the cases for the pairs $(m, N): m=2, N=10$, $12,14,16,18 ; m=3(k=\boldsymbol{Q}(\sqrt{-3})), N=9,12,15 ; m=4(k=\boldsymbol{Q}(\sqrt{-1}))$, $N=8,12 ; m=6(k=Q(\sqrt{-3})), N=6$. Let $x$ be a $k$-rational point on $Y_{1}(m, N)$. Then there exists an elliptic curve $E$ defined over $k$ with a pair $\left(P_{m}, P_{N}\right)$ or $k$-rational points $P_{m}$ and $P_{N}$ such that $\left\langle P_{m}\right\rangle+\left\langle P_{N}\right\rangle \simeq Z / m Z$ $\times Z / N Z$ and that the isomorphism class containing the pair $\left(E, \pm\left(P_{m}, P_{N}\right)\right)$ represents $x$ [4] VI (3.2). For $1 \neq \sigma \in \mathrm{Gal}(k / Q), x^{\sigma}$ is represented by the pair $\left(E^{\sigma}, \pm\left(P_{m}^{\sigma}, P_{N}^{o}\right)\right)$.

THEOREM (3.1). Let $(m, N)$ be a pair as above and $k$ be any quadratic field. If $X_{1}(m, N)$ is not hyperelliptic (i.e., $X_{1}(m, N) \neq \boldsymbol{P}^{1}$ nor $(m, N) \neq(2,10)$, $(2,12))$, then $Y_{1}(m, N)(k)=\phi$.

Proof. Let $J_{1}(m, N)$ and $J_{0}(m, N)$ be the jacobian varieties of the modular curves $X_{1}(m, N)$ and $X_{0}(m, N) \simeq X_{0}(m N)$, respectively, and $\pi$ : $X_{1}(m, N) \rightarrow X_{0}(m, N)$ be the natural morphism. Suppose that there is a $k$-rational point $x$ on $Y_{1}(m, N)$. Let $E$ be an elliptic curve defined over $k$ with $k$-rational points $P_{m}$ and $P_{N}$ such that the pair $\left(E, \pm\left(P_{m}, P_{N}\right)\right)$ represents $x$.

Case $m=6(N=6)$ : Let $p$ be a prime of $k=Q(\sqrt{-3})$ lying over the rational prime 7 and put $R=\left(\mathcal{O}_{k}\right)_{(p)}$. Then $(Z / 6 Z)_{/ R} \times(Z / 6 Z)_{/ R} \subset E_{/ R}$ (1.12), so that $\pi(x) \otimes \kappa(p)=C \otimes \kappa(p)$ for a $Q(\sqrt{-3})$-rational cusp $C$. The modular curve $X_{0}(6,6)$ is an elliptic curve and the restriction of scalars $\operatorname{Re}_{\boldsymbol{Q}(\sqrt{-3}) / \boldsymbol{Q}}\left(X_{0}(6,6)_{/ Q(\sqrt{-3})}\right)$ [5] [34] is isogenous over $\boldsymbol{Q}$ to the product $X_{0}(6,6) \times$ $X_{0}(6,6)$. Since $\sharp X_{0}(6,6)(\boldsymbol{Q})<\infty[36]$ table 1 , we see that $\sharp X_{0}(6,6)(\boldsymbol{Q}(\sqrt{-3}))$ $<\infty$. Then $\pi(x)=C(1,11)$, which is a contradiction.

Case $m=4(N=8,12)$ : In both cases for $N=8$ and $12, \pi(x) \otimes \kappa(p)$ $=C \otimes \kappa(p)$ for a prime $p$ of $k=\boldsymbol{Q}(\sqrt{-1})$ lying over the rational prime 5 and for $k$-rational cusps $C(1.12)$. Let $\pi^{\prime}: X_{0}(4,12) \rightarrow X_{0}(2,12)$ be the natural morphism. The modular curves $X_{0}(4,8)$ and $X_{0}(2,12)$ are elliptic curves and $\sharp X_{0}(4,8)(\boldsymbol{Q}(\sqrt{-1})), \sharp X_{0}(2,12)(\boldsymbol{Q}(\sqrt{-1}))$ are finite (1.15) [36] table 1 . Then the same argument as in the proof for $m=6$ gives a contradiction. 
Case $m=3(N=9,12,5)$ : In all the cases for $N=9,12$ and 15 , $\pi(x) \otimes \kappa(p)=C \otimes \kappa(p)$ for a prime $p$ of $k=\boldsymbol{Q}(\sqrt{-3})$ lying over the rational prime 7 and for $k$-rational cusps $C(1.12)$. The modular curves $X_{0}(3,9)$ and $X_{0}(3,12)$ are elliptic curves $/ \boldsymbol{Q}$ with complex multiplication $\mid \boldsymbol{Q}(\sqrt{-3})$, so the restriction of scalars $\operatorname{Re}_{\boldsymbol{Q}(\sqrt{-3}) / Q}\left(X_{0}(3, N)_{/ Q(\sqrt{-3})}\right)(N=9,12)$ are isogenous over $\boldsymbol{Q}$ to the products $X_{0}(3, N) \times X_{0}(3, N)$. Further $\operatorname{Re}_{\boldsymbol{Q}(\sqrt{-3}) / \boldsymbol{Q}}\left(X_{0}(45)_{/ \boldsymbol{Q}(\sqrt{-3})}\right)$ is isogenous over $\boldsymbol{Q}$ to a product $X_{0}(45)$ and an elliptic curve with conductor 15 (1.15) [36] table 1. Then $\sharp X_{0}(3 N)(\boldsymbol{Q}(\sqrt{-3}))$ $<\infty$ for $N=9,12$ and 15 [36] table 1 . The same argument as above gives contradictions.

Case $m=2(N=14,16,18)$ :

$N=14:$ The modular curve $X_{0}(2,14) \simeq X_{0}(28)$ has the hyperelliptic involution $w_{7}$ (see [36] table 5). Let $p$ be a prime of $k$ lying over the rational prime 3. Then $\pi(x) \otimes \kappa(p)=C \otimes \kappa(p), \pi\left(x^{\sigma}\right) \otimes \kappa(p)=C_{\sigma} \otimes \kappa(p)$ for $\boldsymbol{Q}$-rational cusps $C$ and $C_{\sigma}$. These cusps $C, C_{\sigma}$ are represented by $\left(G_{m} \times Z / 14 Z, A_{2}, A_{14}\right)$ and $\left(G_{m} \times Z / 14 Z, B_{2}, B_{14}\right)$ such that $A_{14} \supset\{1\} \times 2 Z / 14 Z$ and $B_{14} \supset\{1\} \times$ $2 Z / 14 Z$ (1.12). Let $i(x)=\operatorname{cl}\left((x)+\left(x^{\sigma}\right)-(C)-\left(C_{\sigma}\right)\right)$ be the $\boldsymbol{Q}$-rational section of $J_{0}(2,14)_{/ Z}$. Then $i(x)=0$ and ${ }^{r}(x)+\left(x^{\sigma}\right) \sim(C)+\left(C_{o}\right)$, since $\sharp J_{0}(2,14)(\boldsymbol{Q})<\infty(1.4)(1.13)$. But as noted as above, $w_{7}(C) \neq C_{\sigma}$.

$N=16$ : Let $\gamma$ be a generator of the covering group of $X_{1}(32) \rightarrow X_{0}(32)$. Then $Y=X_{1}(32) /\left\langle\gamma^{4}\right\rangle \simeq X_{1}(2,16)$ and $\sharp J(Y)(Q)<\infty(1.4)$. Let $p$ be a prime of $k$ lying over the rational prime 3. Then $x \otimes k(p)=C \otimes \kappa(p), x^{\sigma} \otimes \kappa(p)$ $=C_{\sigma} \otimes \kappa(p)$ for $\boldsymbol{Q}$-rational cusps $C$ and $C_{\sigma}$ (1.12). Considering the $\boldsymbol{Q}$ rational section $i(x)=\operatorname{cl}\left((x)+\left(x^{\sigma}\right)-(C)-\left(C_{\sigma}\right)\right)$ of $J_{1}(2,16)_{/ z}$, we get the relation $(x)+\left(x^{\sigma}\right) \sim(C)+\left(C_{\sigma}\right)$. But $X_{1}(2,16)$ is not hyperelliptic $1(1.7)$.

$N=18$ : Let $p$ be a prime of $k$ lying over the rational prime 5 and put $R=\left(\mathcal{O}_{k}\right)_{(p)}$. By the condition $Z / 2 Z \times Z / 18 Z \subset E(k), E_{/ R} \otimes k(p)=G_{m} \times Z / 18 n Z$ for an integer $n \geqq 1$ (1.12). Then $x \otimes \kappa(p)=C \otimes \kappa(p), x^{\sigma} \otimes \kappa(p)=C_{\sigma} \otimes \kappa(p)$ for $Q$-rational cusps $C$ and $C_{\sigma}$. These cusps $C$ and $C_{\sigma}$ are represented respectively by $\left(G_{m} \times Z / 18 Z, P_{2}, \pm P_{18}\right),\left(G_{m} \times Z / 18 Z, Q_{2}, \pm Q_{18}\right)$, where $P_{n}$, $Q_{n}$ are points of order $n$ such that $P_{18}, Q_{18} \in \mu_{2} \times Z / 18 Z$ (see loc. cit.). Denote also by $x, x^{\sigma}, C$ and $C_{\sigma}$ the images of $x, x^{\sigma}, C$ and $C_{\sigma}$ under the natural morphism of $X_{1}(2,18)$ to $X_{1}(18)$ :

$$
\left(F, B_{2}, \pm B_{18}\right) \longmapsto\left(F, \pm B_{18}\right) \text {. }
$$

Let $i(x)=\operatorname{cl}\left((x)+\left(x^{\sigma}\right)-(C)-\left(C_{\sigma}\right)\right)$ be the $Q$-rational section of $J_{1}(18)_{/ Z}$. 
Since $\sharp J_{1}(18)(\boldsymbol{Q})<\infty(1.4), i(x)=0$ and $(x)+\left(x^{a}\right) \sim(C)+\left(C_{\sigma}\right)$. The modular curve $X_{1}(18)$ has the hyperelliptic involution $\gamma=w_{2}[5]$ :

$$
\left(F, \pm Q_{18}\right) \longmapsto\left(F /\left\langle Q_{2}\right\rangle, \pm\left(Q_{2}^{\prime}+5 Q_{18}\right) \bmod \left\langle Q_{2}\right\rangle\right)
$$

where $Q_{2}, Q_{2}^{\prime}$ are points of order 2 with $Q_{2} \in\left\langle Q_{18}\right\rangle$ and $Q_{2}^{\prime} \notin\left\langle Q_{18}\right\rangle$. Then $x^{\sigma}=\lambda(x)$, so there exists an isomorphism $\lambda(/ C)$

$$
\lambda:\left(E^{\sigma}, \pm P_{18}^{\sigma}\right) \stackrel{\sim}{\longrightarrow}\left(E /\left\langle 9 P_{18}\right\rangle, \pm\left(P^{\prime}+5 P_{18}\right) \bmod \left\langle 9 P_{18}\right\rangle\right),
$$

where $P^{\prime}$ is a point of order 2 not contained in $\left\langle P_{18}\right\rangle$. Since $x \otimes \kappa(p)$ is a cusp, $\lambda$ is defined over a quadratic extension $K$ of $k$ and $\lambda^{\tau}= \pm \lambda$ for $1 \neq \tau \in \operatorname{Gal}(K / k)$. Then $\lambda\left(P_{18}^{\sigma}\right)=\varepsilon\left(P^{\prime}+5 P_{18}\right) \bmod \left\langle 9 P_{18}\right\rangle$ for $\varepsilon= \pm 1$, and it is $k$-rational. Noting that all the 2-torsion points on $E$ are defined over $k$, we see that $\lambda^{\tau}\left(P_{18}^{\sigma}\right)=\left(\lambda\left(P_{18}^{\sigma \tau}\right)\right)^{\tau}=\left(\lambda\left(P_{18}^{\sigma}\right)\right)^{\tau}=\lambda\left(P_{18}^{\tau}\right)$, Thus $\lambda^{\tau}=\lambda$ and $\lambda$ is defined over $k$. Then $\lambda$ induces the isomorphism

$$
\lambda:\left(E^{\sigma}, P_{2}^{o}, P_{18}^{o}\right) \stackrel{\sim}{\longrightarrow}\left(E /\left\langle 9 P_{18}\right\rangle, \lambda\left(P_{2}^{o}\right), \varepsilon\left(P^{\prime}+5 P_{18}^{o}\right) \bmod \left\langle 9 P_{18}\right\rangle\right) .
$$

Let $\mu: E \rightarrow E /\left\langle 9 P_{18}\right\rangle$ be the natural morphism and put $B=\lambda^{-1}\left\{0, \lambda\left(P_{2}^{\sigma}\right)\right\}$. Then $B \neq E_{2}$, so that $B$ is a cyclic subgroup of order 4 defined over $k$. Put $A^{\prime}=\left\langle P^{\prime}+2 P_{18}\right\rangle$ and let $y, y^{\sigma}$ be the $k$-rational points on $X_{0}(4,18) \simeq$ $X_{0}(72)$ represented by the triples $\left(E, B, A^{\prime}\right)$ and $\left(E^{\sigma}, B^{\sigma}, A^{\prime \sigma}\right)$, respectively. Noting that $B \nexists P^{\prime}$ and $B \in 9 P_{18}$, we see that $y \otimes \kappa(p)=C^{\prime} \otimes \kappa(p)$ and $y^{\sigma} \otimes \kappa(p)=C_{o}^{\prime} \otimes \kappa(p)$ for $\boldsymbol{Q}$-rational cusps $C$ and $C_{\sigma}$ (1.12). The remaining part of the proof is the same as that for the case $X_{1}(36)$.

In the rest of this section, we give examples of quadratic fields $k$ such that $Y_{1}(2, N)(k)=\phi$ for $N=10$ and 12 .

Example (3.2). For $N=10$ and $12, X_{1}(2, N)$ are elliptic curves. Let $p$ be a prime of $k$ lying over the rational prime 3 . Then for a $k$-rational point $x$ on $X_{1}(2, N)(N=10,12), \pi(x) \otimes \kappa(p)=C \otimes \kappa(p)$ for a $Q$-rational cusp $C(1.12)$, where $\pi: X_{1}(2, N) \rightarrow X_{0}(2, N)$ is the natural morphism. Set an assumption: $\sharp J_{0}(2, N)(k)<\infty$, and the rational prime 3 is unramified in $k$ or $3 \nmid \# J_{0}(2, N)(k)$. Under this assumption, the same argument as in the proof for $m=6,4$ and 3 (in (3.1)) shows that $Y_{1}(2, N)(k)=\phi$.

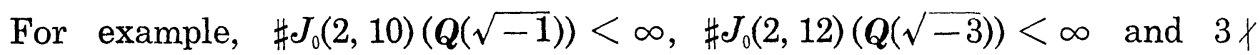
$\sharp J_{0}(2,12)(\boldsymbol{Q}(\sqrt{-3}))(1.15)[36]$ table $1,3,5$. 


\section{REFERENCES}

[1] N. Authaud, On Birch and Swinnerton-Dyer conjecture for elliptic curves with complex multiplication, Compositio Math., 37, 2 (1978), 209-232.

[2] A. O. L. Atkin and J. Lehner, Hecke operators on $\Gamma_{0}(m)$, Math. Ann., 185 (1972), 134-160.

[ 3 ] J. Coates and A. Wiles, On the conjecture of Birch and Swinnerton Dyer, Invent. Math., 39 (1977), 223-251.

[4] P. Deligne and M. Rapoport, Les schémas de modules de courbes elliptique, Proceedings of the International Summer School on Modular functions of one variable, vol. II, Lecture Notes in Math., 349, Springer-Verlag, Berlin-Heidelberg-New York (1973).

[ 5 ] A. Grothendieck, Fondements de la géométrie algébrique, Sèm. Bourbaki, 19571962.

[6] H. Hasse, Über die Klassenzahl Abelscher ZahlKörper (1952), Akademie-Verlag GmbH., Berlin.

[ 7 ] N. Ishii and F. Momose, Hyperelliptic modular curves, to appear.

[ 8 ] M. A. Kenku, Certain torsion points on elliptic curves defined over quadratic fields, J. London Math. Soc. (2) 19 (1979), 233-240.

[9] - The modular curve $X_{0}(39)$ and rational isogeny, Math. Proc. Cambridge Philos. Soc., 85 (1979), 21-23.

[10] - The modular curves $X_{0}(65)$ and $X_{0}(91)$ and rational isogeny, Math. Proc. Cambridge Philos. Soc., 87 (1980), 15-20.

[11] - The modular curve $X_{0}(169)$ and rational isogeny, J. London Math. Soc. (2), 22 (1981), 239-244.

[12] - On the modular curves $X_{0}(125), X_{1}(25)$ and $X_{1}(49)$, J. London Math. Soc. (2), 23 (1981), 415-427.

[13] — Rational torsion points on elliptic curves defined over quadratic fields, to appear.

[14] D. Kubert, Universal bounds on the torsion points of elliptic curves, Proc. London Math. Soc. (3), 33 (1976), 193-237.

[15] Yu. I. Manin, The p-torsion of elliptic curves is uniformally bounded, Math. USSRIzvestija, 3 (1969), 433-438.

[16] - Parabolic points and zeta-functions of modular curves, Math. USSR-Izvestija, 6 (1972), 19-64.

[17] B. Mazur, Rational points on modular curves, Proceedings of Conference on Modular Functions held in Bonn, Lecture Notes in Math. 601, Springer-Verlag, Berlin-Heiderberg-New York (1977).

[18] — Rational isogenies of prime degree, Invent. Math., 44 (1978), 129-162.

[19] B. Mazur and J. Tate, Points of order 13 on elliptic curves, Invent. Math., 22 (1973), 41-49.

[20] J. F. Mestre, Points rationnels de la courbe modulaire $X_{0}(169)$, Ann. Inst. Fourier, 30, 2 (1980), 17-27.

[21] J. S. Milne, On the arithmetic of abelian varieties, Invent. Math., 17 (1972), $177-190$.

[22] F. Momose, Rational points on the modular curves $X_{\text {split }}(p)$, Compositio Math., 52 (1984), 115-137.

[23] $-p$-torsion points on elliptic curves defined over quadratic fields, Nagoya Math. J., 96 (1984), 139-165.

[24] A. Ogg, Rational points on certain elliptic modular curves, Proc. Symposia in Pure Math. XXIX, AMS, (1973) 221-231. 
[25] —- Hyperelliptic modular curves, Bull. Soc. Math. France, 102 (1974), 449-462.

[26] _- Diophantine equations and modular forms, Bull. AMS, 81 (1975), 14-27.

[27] F. Oort and J. Tate, Group schemes of prime order, Ann. Scient. Ec. Norm. Sup. serie 4, 3 (1970), 1-21.

[28] M. Raynaud, Schémas en groupes de type $(p, \cdots, p)$, Bull. Soc. Math. France, 102 (1974), 241-280.

[29] K. Rubin, Congruences for special values of $L$-functions of elliptic curves with complex multiplication, Invent. Math., 71 (1983), 339-364.

[30] J. P. Serre, Propriétés galoissiennes des points d'ordre fini des courbes elliptiques, Invent. Math., 15 (1972), 259-331.

[31] — $p$-torsion des courbes elliptiques (d'après Y. Manin), Sèm. Boubaki 1969/70, 281-294, Lecture Notes in Math. 180, Springer-Verlag, Berlin-Heidelberg-New York (1971).

[32] G. Shimura, Introduction to the arithmetic theory of automorphic functions, Publ. Math. Soc. Japan 11, Iwanami Shoten, Tokyo-Princeton Univ. Press, Princeton, N.J.

[33] - On elliptic curves with complex multiplication as factors of the jacobians of modular function fields, Nagoya Math. J., 43 (1971), 199-208.

[34] A. Weil, Adèles and algebraic groups, Lecture Notes, Inst. for Advanced Study, Princeton, N.J.

[35] J. Yu, A cuspidal class number formula for the modular curves $X_{1}(N)$, Math. Ann., 252 (1980), 197-216.

[36] Modular functions of one variable IV (ed. by B. J. Birch and W. Kuyk), Lecture Notes in Math., 476, Springer-Verlag, Berlin-Heidelberg-New York (1975).

\author{
M. A. Kenku \\ Department of Mathematics \\ Faculty of Science \\ University of Lagos \\ Lagos, Nigeria \\ F. Momose \\ Department of Mathematics \\ Chuo University \\ 1-13-27 Kasuga, Bunkyo-ku \\ Tokyo 112, Japan
}

